\title{
A Mathematical Justification for the Herman-Kluk Propagator
}

\author{
Torben SWART and Vidian ROUSSE \\ Freie Universität Berlin
}

October 25, 2018

\begin{abstract}
A class of Fourier Integral Operators which converge to the unitary group of the Schrödinger equation in semiclassical limit $\varepsilon \rightarrow 0$ is constructed. The convergence is in the uniform operator norm and allows for an error bound of order $O\left(\varepsilon^{1-\rho}\right)$ for Ehrenfest timescales, where $\rho$ can be made arbitrary small. For the shorter times of order $O(1)$, the error can be improved to arbitrary order in $\varepsilon$. In the chemical literature the approximation is known as the Herman-Kluk propagator.
\end{abstract}

\section{Introduction}

We study approximate solutions of the semiclassical time-dependent Schrödinger equation

$$
i \varepsilon \frac{d}{d t} \psi^{\varepsilon}(t)=-\frac{\varepsilon^{2}}{2} \Delta \psi^{\varepsilon}(t)+V(x) \psi^{\varepsilon}(t), \quad \psi^{\varepsilon}(0)=\psi_{0}^{\varepsilon} \in L^{2}\left(\mathbb{R}^{d}, \mathbb{C}\right)
$$

in the semiclassical limit $\varepsilon \rightarrow 0$. The operator $H^{\varepsilon}:=-\frac{\varepsilon^{2}}{2} \Delta+V(x)$ on the right-hand side of (10) is the so-called Hamiltonian, a self-adjoint operator on $L^{2}\left(\mathbb{R}^{d}, \mathbb{C}\right)$. It is well-known that the solution of (1) can be written as

$$
\psi^{\varepsilon}(t)=e^{-\frac{i}{\varepsilon} H^{\varepsilon} t} \psi_{0}^{\varepsilon},
$$

where the group of unitary operators $e^{-\frac{i}{\varepsilon} H^{\varepsilon} t}$ is defined by the spectral theorem. The semiclassical parameter $\varepsilon$ may be thought of as the quantum of action $\hbar$, but there are also situations, where $\varepsilon$ has a different meaning. One example is provided by Born-Oppenheimer molecular dynamics, where equation (1) describes the semiclassical motion of the nuclei of a molecule in the case of well-separated electronic energy surfaces and $\varepsilon$ is the square root of the ratio of the electronic mass and the average nuclear mass. In this case, the $\varepsilon$ in front of the timederivative in (10) is due to a rescaling of time $\widetilde{t}=t / \varepsilon$. This particular choice, the so-called "distinguished limit" (see Co68) produces the most interesting results in the semiclassical limit $\varepsilon \rightarrow 0$.

To formulate our main result, we introduce the following class of Fourier Integral Operators (FIOs):

$$
\mathcal{I}^{\varepsilon}\left(\kappa^{t} ; u\right) \varphi(x):=\frac{1}{(2 \pi \varepsilon)^{3 d / 2}} \int_{\mathbb{R}^{3 d}} e^{\frac{i}{\varepsilon} \Phi^{\kappa^{t}}(x, y, q, p)} u(x, y, q, p) \varphi(y) d q d p d y,
$$


where

- $\kappa^{t}(q, p)=\left(X^{\kappa^{t}}(q, p), \Xi^{\kappa^{t}}(q, p)\right)$ is a $C^{1}$-family of canonical transformations of the classical phase space $T^{*} \mathbb{R}^{d}=\mathbb{R}^{d} \times \mathbb{R}^{d}$,

- $S^{\kappa^{t}}(q, p)$ is the associated classical action

$$
S^{\kappa^{t}}(q, p)=\int_{0}^{t}\left[\frac{d}{d t} X^{\kappa^{\tau}}(q, p) \cdot \Xi^{\kappa^{\tau}}(q, p)-\left(h \circ \kappa^{\tau}\right)(q, p)\right] d \tau,
$$

- the complex-valued phase function is given by

$$
\begin{aligned}
\Phi^{\kappa^{t}}(x, y, q, p)= & S^{\kappa^{t}}(q, p)+\Xi^{\kappa^{t}}(q, p) \cdot\left(x-X^{\kappa^{t}}(q, p)\right)-p \cdot(y-q) \\
& +\frac{i}{2}\left|x-X^{\kappa^{t}}(q, p)\right|^{2}+\frac{i}{2}|y-q|^{2}
\end{aligned}
$$

- and the symbol $u$ is a smooth complex-valued function which is bounded with all its derivatives.

For this class of operators, the authors previously established an $L^{2}$-boundedness result, see RoSw07. The central result of this paper reads

Theorem. Let $e^{-\frac{i}{\varepsilon} H^{\varepsilon} t}$ be the propagator defined by the time-dependent Schrödinger equation (10) on the time-interval $[-T, T]$ with subquadratic potential $V \in$ $C^{\infty}\left(\mathbb{R}^{d}, \mathbb{R}\right)$, i.e. $\sup _{x \in \mathbb{R}^{d}}\left|\partial_{x}^{\alpha} V(x)\right|<\infty$ for all $\alpha \in \mathbb{N}^{d}$ with $|\alpha| \geq 2$.

Then

$$
\sup _{t \in[-T, T]}\left\|e^{-\frac{i}{\varepsilon} H^{\varepsilon} t}-\mathcal{I}^{\varepsilon}\left(\kappa^{t} ; u\right)\right\|_{L^{2} \rightarrow L^{2}} \leq C(T) \varepsilon,
$$

where $\kappa^{t}=\left(X^{\kappa^{t}}, \Xi^{\kappa^{t}}\right)$ and $u$ are uniquely given as

- the flow associated to the classical Hamiltonian $h(x, \xi)=\frac{1}{2}|\xi|^{2}+V(x)$

$$
\begin{aligned}
\frac{d}{d t} X^{\kappa^{t}}(q, p) & =\Xi^{\kappa^{t}}(q, p) & X^{\kappa^{0}}(q, p) & =q \\
\frac{d}{d t} \Xi^{\kappa^{t}}(q, p) & =-\nabla V\left(X^{\kappa^{t}}(q, p)\right) & \Xi^{\kappa^{0}}(q, p) & =p
\end{aligned}
$$

and

- the solution of the Cauchy-problem

$$
\begin{aligned}
\frac{d}{d t} u(t, q, p) & =\frac{1}{2} u(t, q, p) \operatorname{tr}\left(\mathcal{Z}^{-1}\left(F^{\kappa^{t}}(q, p)\right) \frac{d}{d t} \mathcal{Z}\left(F^{\kappa^{t}}(q, p)\right)\right) \\
u(0, q, p) & =2^{d / 2} .
\end{aligned}
$$

The $\mathbb{C}^{d \times d}$-valued function

$$
\begin{aligned}
& \mathcal{Z}\left(F^{\kappa^{t}}(q, p)\right)=(i \text { id } \quad \text { id }) F^{\kappa^{t}}(q, p)^{\dagger}\left(\begin{array}{c}
-i \text { id } \\
\text { id }
\end{array}\right) \\
& =X_{q}^{\kappa^{t}}(q, p)-i X_{p}^{\kappa^{t}}(q, p)+i \Xi_{q}^{\kappa^{t}}(q, p)+\Xi_{p}^{\kappa^{t}}(q, p),
\end{aligned}
$$


depends on elements of the transposed Jacobian

$$
F^{\kappa^{t}}(q, p)^{\dagger}=\left(\begin{array}{cc}
X_{q}^{\kappa^{t}}(q, p) & \Xi_{q}^{\kappa^{t}}(q, p) \\
X_{p}^{\kappa^{t}}(q, p) & \Xi_{p}^{\kappa^{t}}(q, p)
\end{array}\right)
$$

of $\kappa^{t}$ with respect to $(q, p)$.

The equation for $u$ is easily solved. Its solution is the so-called Herman-Kluk prefactor

$$
u(t, q, p)=\left(\operatorname{det} \mathcal{Z}\left(F^{\kappa^{t}}(q, p)\right)\right)^{\frac{1}{2}},
$$

where the branch of the square root is chosen by continuity in time starting from $t=0$. We presented a simplified version of our main result. Theorem 2 will essentially add three central aspects. First, we will state it for more general Hamilton operators, namely certain Weyl-quantised pseudodifferential operators. Second, for the Ehrenfest-timescale $T(\varepsilon)=C_{T} \log \left(\varepsilon^{-1}\right)$ the result still holds with a slightly weaker bound. Third, the error estimate can be improved to $\varepsilon^{N}$, where $N$ is arbitrary large by adding a correction of the form $\sum_{n=1}^{N-1} \varepsilon^{n} u_{n}$ to $u$. As $u$, the $u_{n}$ are solutions of explicitly solvable Cauchy-problems.

Whereas there is an abundant number of works on Fourier Integral Operators in the mathematical literature, only few of them discuss the relation between FIOs and the time-dependent Schrödinger-equation. The first works which apply FIOs with real-valued phase function to this problem are [KiKu81] and Ki82]. In this case one has to deal with the boundary value problem

$$
\text { Given } x, y \in \mathbb{R}^{d} \text {, find } p \text { such that } X^{\kappa^{t}}(y, p)=x \text {. }
$$

To get uniqueness for its solution one has either to restrict to short times $t$ or to impose very strong restrictions on the potential. The same problems are met in Fu79, where Fujiwara applies a related class of operators without integral in the oscillatory kernel to the Schrödinger equation to justify the time-slicing approach for Feynman's path integrals.

The avoidance of this problem is the major advantage of complex-valued phase functions. In the non-semiclassical setting, Tataru shows in Ta04 that the unitary group of time evolution is an FIO with complex-valued phase function (different from (3) ). He also establishes that the simpler choice $u(t, q, p)=2^{d / 2}$ leads to a parametrix for the non-semiclassical Schrödinger equation.

A class of operators related to (2) is used in the works [LaSi00 and $\mathrm{Bu02}$ for the construction of approximate solutions of the semiclassical time-dependent Schrödinger equation. In their case, the kernel consists of an integral over the momentum space in contrast to the phase-space integral in our expression

$$
\left(\widetilde{\mathcal{I}}^{\varepsilon}\left(\kappa^{t} ; \widetilde{u}\right) \psi\right)(x)=\frac{1}{(2 \pi \varepsilon)^{d}} \int_{T^{*} \mathbb{R}^{d}} e^{\frac{i}{\varepsilon} \Phi^{\kappa^{t}}(x, y, y, p)} \widetilde{u}(t, y, p) \psi(y) d p d y
$$

Moreover, these works only allow compactly supported symbols, which enforces the truncation of the Hamiltonian in momentum. Finally there is the work of Bily and Robert BiRo01, which treats the so-called Thawed Gaussian Approximation discussed below.

In contrast to the mathematical literature connecting time-dependent Schrödinger equation and Fourier Integral Operators, there is an abundant number of papers in chemical journals on this topic. Nevertheless, the focus is mainly 
put on three approximations: the "Thawed Gaussian Approximation" (TGA), the "Frozen Gaussian Approximation" (FGA) and the Herman-Kluk expression. Confusingly, in the chemical literature both TGA and FGA do not only refer to specific algorithms but they are also used to describe whole classes of approximations. For example, the Herman-Kluk approximation is sometimes considered as an FGA, whereas the TGA refers both to the time-evolution of a coherent state and a Fourier Integral Operator. We give a short formal discussion of the most important methods in the rest of this introduction hinting to related rigorous results.

The starting point is the following identity, which holds for $\psi \in L^{2}\left(\mathbb{R}^{d}, \mathbb{C}\right)$ :

$$
\psi(x)=\frac{1}{(2 \pi \varepsilon)^{d}} \int_{T^{*} \mathbb{R}^{d}} g_{(q, p)}^{\varepsilon}(x)\left\langle g_{(q, p)}^{\varepsilon}, \psi\right\rangle d q d p,
$$

where

$$
g_{(q, p)}^{\varepsilon}(x)=\frac{1}{(\pi \varepsilon)^{d / 4}} e^{-|x-q|^{2} / 2 \varepsilon} e^{i p \cdot(x-q) / \varepsilon}
$$

denotes the coherent state centered at $(q, p)$ in phase space $T^{*} \mathbb{R}^{d}$. Within the chemical community, equation (4) is heuristically explained as an "expansion in an overcomplete set of Gaussians", but the equality can be made rigorous with help of the FBI-transform, consider Ma02]. Applying the unitary group of (10) to expression (4), one gets the formal equality

$$
\left(e^{-\frac{i}{\varepsilon} o p^{\varepsilon}(h) t} \psi_{0}^{\varepsilon}\right)(x)=\frac{1}{(2 \pi \varepsilon)^{d}} \int_{T^{*} \mathbb{R}^{d}}\left(e^{-\frac{i}{\varepsilon} o p^{\varepsilon}(h) t} g_{(q, p)}^{\varepsilon}\right)(x)\left\langle g_{(q, p)}^{\varepsilon}, \psi_{0}^{\varepsilon}\right\rangle d q d p .
$$

Hence, one expects an approximation to the solution of (1) if the following approximate expression for the time-evolution of coherent states is used in (6)

$$
\begin{aligned}
& \left(e^{-\frac{i}{\varepsilon} o p^{\varepsilon}(h) t} g_{(q, p)}^{\varepsilon}\right)(x) \approx \frac{1}{(\pi \varepsilon)^{d / 4}}\left[\operatorname{det}\left(X_{q}^{\kappa^{t}}(q, p)+i X_{p}^{\kappa^{t}}(q, p)\right)\right]^{-\frac{1}{2}} \\
& \quad \times e^{\frac{i}{\varepsilon} S^{\kappa^{t}}(q, p)} e^{-\left(x-X^{\kappa^{t}}(q, p)\right) \cdot \Theta^{\kappa^{t}}(q, p)\left(x-X^{\kappa^{t}}(q, p)\right) / 2 \varepsilon} e^{i \Xi^{\kappa^{t}}(q, p) \cdot\left(x-X^{\kappa^{t}}(q, p)\right) / \varepsilon}
\end{aligned}
$$

with

$$
\Theta^{\kappa^{t}}(q, p)=-i\left(\Xi_{q}^{\kappa^{t}}(q, p)+i \Xi_{p}^{\kappa^{t}}(q, p)\right)\left(X_{q}^{\kappa^{t}}(q, p)+i X_{p}^{\kappa^{t}}(q, p)\right)^{-1} .
$$

In the chemical literature (7) was first derived in [He75]. For rigorous mathematical results consider Ha85, Ha98 or CoRo97. As the coherent state changes its width, expression (7) and the resulting operator were baptised "Thawed Gaussian Approximation".

However, it turns out numerically (see e.g. the computations in HaRoGr04) that more accurate approximations are obtained if one drops the time-dependent spreading and uses expressions like (2). In the simplest case, the symbol $u \equiv 1$ is held constant in $t, q$ and $p$. This approximation is known as the "Frozen Gaussian Approximation" and holds only for times of order $O(\varepsilon)$, see the remark after Theorem 2, To get to the longer times of order $O(1)$, the more sophisticated choice of $u(t, q, p)$ as the Herman-Kluk prefactor is needed, see [HeKl84] for the original work and Ka94 and Ka06] for works, which are methodically related to our presentation. Moreover, the latter of them presents the first derivation of the higher order corrections. 


\section{Organisation of the paper and notation}

The paper is organised in the following way. Section 2 will set the stage for the discussion of our approximation. Here we will recall central definitions and results on Fourier Integral Operators, first and foremost their definition and well-definedness on the functions of Schwartz class as well as their bound as operators acting on $L^{2}\left(\mathbb{R}^{d}, \mathbb{C}\right)$, see Definition 6] and Theorem 11. Most of the results of this section can be found in RoSw07. and we refer the reader to that paper for a more detailed discussion and motivation of them. In Section 3 we will prove results on the composition of Weyl-quantised pseudo-differential operators and Fourier Integral Operators, see Proposition 2. Moreover, we will investigate the time-derivative of a $C^{1}$-family of Fourier Integral Operators in Proposition 3. These results will lead to our main result, which we will state in Theorem 2 .

We close this introduction by a short discussion of the notation. Throughout this paper, we will use standard multiindex notation. Vectors will always be considered as column vectors. The inner product of two vectors $a, b \in \mathbb{R}^{d}$ will be denoted as $a \cdot b=\sum_{j=1}^{d} a_{j} b_{j}$ and extended to vectors $a, b \in \mathbb{C}^{d}$ by the same formula. The transpose of a matrix $A$ will be $A^{\dagger}$, whereas $A^{*}:=\bar{A}^{\dagger}$ denotes the adjoint and finally $e_{j}$ will stand for the $j$ th canonical basis vector of $\mathbb{R}^{d}$ or $\mathbb{C}^{d}$. For a differentiable mapping $F \in C^{1}\left(\mathbb{R}^{d}, \mathbb{C}^{d}\right)$, we will use both $\left(\partial_{x} F\right)(x)$ and $F_{x}(x)$ for the transpose of its Jacobian at $x$, i.e. $\left(\left(\partial_{x} F\right)(x)\right)_{j k}=\left(F_{x}(x)\right)_{j k}=$ $\left(\partial_{x_{j}} F_{k}\right)(x)$. This leads to the identity $\partial_{x}(F \cdot G)=G_{x} F+F_{x} G$ for $F, G \in$ $C^{1}\left(\mathbb{R}^{d}, \mathbb{C}^{d}\right)$. The Hessian matrix of a mapping $F \in C^{2}\left(\mathbb{R}^{d}, \mathbb{C}\right)$ will be denoted by $\operatorname{Hess}_{x} F(x)$.

For the sake of better readability of the formulae, we will be somewhat sloppy with respect to the distinction between functions and their values. As a crucial example, we will write $\left(x-X^{\kappa}(q, p)\right) v$ for the function $(x, y, q, p) \mapsto(x-$ $\left.X^{\kappa}(q, p)\right) v(x, y, q, p)$.

When dealing with canonical transformations, we introduce the following notations for a complex linear combinations of the components:

$$
\begin{aligned}
& Z^{\kappa}(q, p):=\left(\Theta^{x}\right)^{\frac{1}{2}} X^{\kappa}(q, p)+i\left(\Theta^{x}\right)^{-\frac{1}{2}} \Xi^{\kappa}(q, p) \\
& \bar{Z}^{\kappa}(q, p):=\left(\Theta^{x}\right)^{\frac{1}{2}} X^{\kappa}(q, p)-i\left(\Theta^{x}\right)^{-\frac{1}{2}} \Xi^{\kappa}(q, p) .
\end{aligned}
$$

We want to point out that $\bar{Z}^{\kappa}(q, p)$ is not the complex conjugate of $Z^{\kappa}(q, p)$ for non-real matrices $\Theta^{x}$. The matrix square root of a positive definite matrix will always be chosen as the unique positive definite square root. We want to point out that both the determinant of this matrix-square root and the square root of a determinant will appear in this paper.

We define $z:=\Theta^{y} q+i p, \partial_{z}:=\left(\Theta^{y}\right)^{-1} \partial_{q}-i \partial_{p}$ and

$$
\operatorname{div}_{z} X(q, p)=\sum_{k=1}^{d}\left(\Theta^{y}\right)_{j k}^{-1} \partial_{q_{k}} X_{j}(q, p)-i \sum_{j=1}^{d} \partial_{p_{j}} X_{j}(q, p)
$$

for functions $X \in C^{1}\left(\mathbb{R}^{2 d}, \mathbb{C}^{d}\right)$, regardless whether they are row or column vectors. With these definitions the identity $\operatorname{div}_{z} X(q, p)=\operatorname{tr} X_{z}(q, p)$ still holds. Finally, we mention that the expression $\frac{d}{d t} X^{\kappa^{(t, s)}}(q, p) \cdot \Xi^{\kappa^{(t, s)}}(q, p)$ denotes the inner product of $\frac{d}{d t} X^{\kappa^{(t, s)}}(q, p)$ and $\Xi^{\kappa^{(t, s)}}(q, p)$. 


\section{Acknowledgement}

The authors want to thank Caroline Lasser for many profitable discussions and valuable comments.

\section{Canonical Transformations and Fourier Inte- gral Operators}

In this section, we specialise the central definitions and results of [RoSw07] to the case of Hamiltonian flows.

\subsection{Symbol classes and canonical transformations}

The definition of our FIOs involves two fundamental objects. One of them is a smooth complex-valued function, the so-called symbol. The following definition deviates from [RoSw07] by the additional $\varepsilon$-dependence.

Definition 1 (Symbol class). Let $\mathbf{m}=\left(m_{j}\right)_{1 \leq j \leq J} \in \mathbb{R}^{J}$ and $\mathbf{d}=\left(d_{j}\right)_{1 \leq j \leq J} \in$ $\mathbb{N}^{J}$. We say that $\left.\left.u:\right] 0,1\right] \times \mathbb{R}^{|\mathbf{d}|} \rightarrow \mathbb{C}^{N}$ is a symbol of class $S[\mathbf{m} ; \mathbf{d}]$, if there is $\varepsilon_{0}<1$, such that $u^{\varepsilon} \in C^{\infty}\left(\mathbb{R}^{|\mathbf{d}|}, \mathbb{C}^{N}\right)$ for all $\varepsilon \leq \varepsilon_{0}$ and the following quantities are finite for any $k \geq 0$

$$
M_{k}^{m}[u]:=\sup _{\varepsilon \leq \varepsilon_{0}|\alpha|=k} \max _{z \in \mathbb{R}^{|\mathbf{d}|}}\left|\prod_{j=1}^{J}\left\langle z_{j}\right\rangle^{-m_{j}} \partial_{z}^{\alpha} u^{\varepsilon}(z)\right|,
$$

where $\langle z\rangle:=\sqrt{1+|z|^{2}}$. We extend this definition by setting

$$
S[+\infty ; \mathbf{d}]=\bigcup_{m_{1} \in \mathbb{R}} \ldots \bigcup_{m_{J} \in \mathbb{R}} S\left[\left(m_{1}, \ldots, m_{J}\right) ; \mathbf{d}\right] .
$$

The second central object in the definition of a Fourier Integral Operator is a canonical transformation of the classical phase space.

Definition 2 (Canonical transformation). Let $\kappa(q, p)=\left(X^{\kappa}(q, p), \Xi^{\kappa}(q, p)\right)$ be a diffeomorphism of $T^{*} \mathbb{R}^{d}=\mathbb{R}^{d} \times \mathbb{R}^{d}$. We represent its differential by the following Jacobian matrix

$$
F^{\kappa}(q, p)=\left(\begin{array}{cc}
X_{q}^{\kappa}(q, p)^{\dagger} & X_{p}^{\kappa}(q, p)^{\dagger} \\
\Xi_{q}^{\kappa}(q, p)^{\dagger} & \Xi_{p}^{\kappa}(q, p)^{\dagger}
\end{array}\right) .
$$

$\kappa$ is said to be a canonical transformation if $F^{\kappa}(q, p)$ is symplectic for any $(q, p)$ in $T^{*} \mathbb{R}^{d}$, i.e.

$$
F^{\kappa}(q, p) \in \mathrm{Sp}(2 d):=\left\{S \in \mathrm{Gl}(2 d) \mid S^{\dagger} J S=J\right\} \quad \text { with } \quad J:=\left(\begin{array}{cc}
0 & \text { id } \\
-\mathrm{id} & 0
\end{array}\right) .
$$

To get good properties for our operators, we need to restrict the class of canonical transformations under consideration. 
Definition 3 (Canonical transformation of class $\mathcal{B}$ ). A canonical transformation $\kappa$ of $T^{*} \mathbb{R}^{d}$ is said to be of class $\mathcal{B}$ if $F^{\kappa} \in S[0 ; 2 d]$. A time-dependent family of canonical transformations $\kappa^{t}$ will be called of class $\mathcal{B}$ in $[-T, T]$ if it is pointwise continuously differentiable with respect to time and we have for all $k \geq 0$

$$
\sup _{t \in[-T, T]} M_{k}^{0}\left[F^{\kappa^{t}}\right]<\infty \quad \text { and } \quad \sup _{t \in[-T, T]} M_{k}^{0}\left[\frac{d}{d t} F^{\kappa^{t}}\right]<\infty .
$$

In particular $F^{\kappa^{t}}$ and $\frac{d}{d t} F^{\kappa^{t}}$ are of class $S[0 ; 2 d]$ pointwise for $t \in[-T, T]$.

We also have to restrict the Hamiltonians we use.

Definition 4. A time-dependent Hamiltonian $h \in C\left(\mathbb{R}, C^{\infty}\left(\mathbb{R}^{2 d}, \mathbb{C}\right)\right)$ is called subquadratic, if

$$
\sup _{-T \leq t \leq T} \sup _{(x, \xi) \in \mathbb{R}^{d} \times \mathbb{R}^{d}}\left\|\partial_{(x, \xi)}^{\alpha} h(t, x, \xi)\right\|_{L^{\infty}}
$$

is finite for all $|\alpha| \geq 2$ and $T>0$. It is called sublinear, if the quantity is finite for all $|\alpha| \geq 1$.

The next result will investigate the relation between classical Hamiltonians and the flows they generate.

Proposition 1. If $h \in C\left(\mathbb{R}, C^{\infty}\left(\mathbb{R}^{2 d}, \mathbb{C}\right)\right)$ is a time-dependent subquadratic Hamiltonian, the Hamiltonian flow $\kappa^{(t, s)}$ generated by $h$,

$$
\frac{d}{d t} \kappa^{(t, s)}=J \nabla_{(x, \xi)} h\left(t, \kappa^{(t, s)}\right), \quad \kappa^{(s, s)}=\mathrm{id}
$$

is a family of canonical transformations of class $\mathcal{B}$ in $[-T, T]$. Moreover, every Hamiltonian flow of class $\mathcal{B}$ is generated by a subquadratic Hamiltonian.

Under the additional assumption $\left\|\partial_{(x, \xi)}^{\alpha} h\right\|_{L^{\infty}\left(\mathbb{R} \times \mathbb{R}^{2 d}\right)}<\infty$ for all $2 \leq|\alpha| \leq$ $n_{0}+2$, we have

$$
\sup _{|t-s|<T(\varepsilon)} M_{k}^{0}\left[F^{\kappa^{(t, s)}}\right] \leq C_{k}\left(2 C_{T}\right)^{k}|\log \varepsilon|^{k} \varepsilon^{-2 K_{0}^{h} C_{T}},
$$

for all $k \leq n_{0}$ on the Ehrenfest timescale $T(\varepsilon)=C_{T} \log \varepsilon^{-1}$, where

$$
K_{k}^{h}(T)=\max _{|\alpha|=k} \sup _{-T \leq t \leq T} \sup _{(x, \xi) \in \mathbb{R}^{d} \times \mathbb{R}^{d}}\left\|\partial_{(x, \xi)}^{\alpha} \operatorname{Hess}_{(x, \xi)} h(t, x, \xi)\right\| .
$$

Proof. The basic identity follows by differentiating (12) with respect to $(q, p)$ :

$$
\frac{d}{d t} F^{\kappa^{(t, s)}}(q, p)=J \operatorname{Hess}_{(x, \xi)} h\left(t, \kappa^{(t, s)}(q, p)\right) F^{\kappa^{(t, s)}}(q, p) .
$$

The fundamental theorem of calculus gives

$$
\begin{aligned}
\left\|F^{\kappa^{(t, s)}}(q, p)\right\| & \leq\left|\int_{s}^{t}\left\|\operatorname{Hess}_{(x, \xi)} h\left(\tau, \kappa^{(\tau, s)}(q, p)\right)\right\|\left\|F^{\kappa^{(\tau, s)}}(q, p)\right\| d \tau\right|+\|\mathrm{id}\| \\
& \leq K_{0}^{h}(T)\left|\int_{s}^{t}\left\|F^{\kappa^{(\tau, s)}}(q, p)\right\| d \tau\right|+1 .
\end{aligned}
$$


Hence, the bound for the Jacobian follows from Gronwall's Lemma:

$$
\left\|F^{\kappa^{(t, s)}}(q, p)\right\| \leq e^{K_{0}^{h}(T)|t-s|} .
$$

For the derivatives we have

$$
\begin{aligned}
& \left\|\partial_{(q, p)}^{\alpha} F^{\kappa^{(t, s)}}(q, p)\right\| \leq\left|\int_{s}^{t} K_{0}^{h}(T)\left\|\partial_{(q, p)}^{\alpha} F^{\kappa^{(\tau, s)}}(q, p)\right\| d \tau\right| \\
& +\left|\int_{s}^{t} \sum_{\beta<\alpha}\left(\begin{array}{c}
\alpha \\
\beta
\end{array}\right)\left\|\partial_{(q, p)}^{\alpha-\beta}\left[\operatorname{Hess}_{(x, \xi)} h\left(\tau, \kappa^{(\tau, s)}(q, p)\right)\right] \partial_{(q, p)}^{\beta} F^{\kappa^{(t, s)}}(q, p)\right\| d \tau\right|,
\end{aligned}
$$

so Gronwall's Lemma provides inductively

$$
\left\|\partial_{(q, p)}^{\alpha} F^{\kappa^{(t, s)}}(q, p)\right\| \leq C_{k}(2 T)^{k} e^{K_{0}^{h}(T)|t-s|},
$$

where $C_{k}$ depends on $K_{l}^{h}(T)$ for $l \leq k$. The result for the Ehrenfest timescale follows by substituting $T(\varepsilon)=C_{T} \log \left(\varepsilon^{-1}\right)$ into this expression.

Now consider a Hamiltonian flow of class $\mathcal{B}$. The identity (13) gives

$$
J\left(\frac{d}{d t} F^{\kappa^{(t, s)}}(q, p)\right) J\left(F^{\kappa^{(t, s)}}(q, p)\right)^{\dagger} J=\operatorname{Hess}_{(q, p)} h\left(t, \kappa^{(t, s)}(q, p)\right)
$$

Hence, $h$ is subquadratic, as $\frac{d}{d t} F^{\kappa^{(t, s)}}$ is of class $S[0 ; 2 d]$ by definition.

\section{Remark 1.}

1. It is easy to show that there is an equivalence between linear Hamiltonian flows and quadratic Hamiltonians. Every quadratic Hamiltonian generates a linear flow and every Hamiltonian linear flow $\kappa^{(t, s)}(q, p)=M(t, s)\left(\begin{array}{l}q \\ p\end{array}\right)$, $M(\cdot, s) \in C^{1}(\mathbb{R}, \operatorname{Sp}(2 d))$ is generated by a quadratic Hamiltonian, namely

$$
h_{M}(t, q, p)=\frac{1}{2}(q p)\left(J\left(\frac{d}{d t} M(t, s)\right) J M^{\dagger}(t, s) J\right)\left(\begin{array}{l}
q \\
p
\end{array}\right) .
$$

2. By estimating the logarithm, we can have a bound of the form

$$
\sup _{|t-s|<T(\varepsilon)} M_{k}^{0}\left[F^{\kappa^{(t, s)}}\right] \leq C_{k}^{\prime}\left(C_{T}\right) \varepsilon^{-\rho\left(C_{T}\right)},
$$

for the Ehrenfest timescale, where $\rho\left(C_{T}\right)<\rho_{0}$ for any $\rho_{0}>0$, if $C_{T}$ is chosen small enough.

3. From now on, all considered canonical transformations are assumed to be of class $\mathcal{B}$.

An important quantity associated with a canonical transformation is the socalled action.

Definition 5 (Action). Let $\kappa(q, p)=\left(X^{\kappa}(q, p), \Xi^{\kappa}(q, p)\right)$ be a canonical transformation of $T^{*} \mathbb{R}^{d}$. A real-valued function $S^{\kappa}$ is called an action associated to $\kappa$ if it fulfills

$$
S_{q}^{\kappa}(q, p)=-p+X_{q}^{\kappa}(q, p) \Xi^{\kappa}(q, p), \quad S_{p}^{\kappa}(q, p)=X_{p}^{\kappa}(q, p) \Xi^{\kappa}(q, p) .
$$


Remark 2.

1. An action associated to a canonical transformation is only defined up to an additive constant. If we consider a time-dependent family of canonical transformations $\kappa^{t}$, we will choose this time-dependent constant such that $S^{\kappa^{t}}(q, p)$ is $C^{1}$ with respect to time.

2. If $\kappa^{(t, s)}$ is induced by a Hamiltonian $h(t, x, \xi)$, the action of classical mechanics

$$
S_{c l}^{\kappa^{(t, s)}}(q, p)=\int_{s}^{t}\left(\frac{d}{d t} X^{\kappa^{\tau}}(q, p) \cdot \Xi^{\kappa^{\tau}}(q, p)-h\left(\tau, \kappa^{(\tau, s)}(q, p)\right)\right) d \tau
$$

is an action in the sense of this definition. In this case, we use the convention $S^{\kappa^{(s, s)}}(q, p)=0$, where $S^{\kappa^{(t, s)}}(q, p)$ is now considered as a function of $t$. We cannot assume $S^{\text {id }}(q, p)=0$, as the case $h(t, x, \xi)=h(t)$ shows.

\subsection{Definition of FIOs and continuity results}

In this section, we will define the operators we will use to approximate of the propagator of the Schrödinger equation.

Definition 6 (Fourier Integral Operator). For $u \in S\left[\left(+\infty, m^{p}\right) ;(3 d, d)\right]$, a Schwartz-class function $\varphi \in \mathcal{S}\left(\mathbb{R}^{d}, \mathbb{C}\right)$ and $n>m^{p}+d$, we define

$$
\begin{aligned}
& {\left[\mathcal{I}^{\varepsilon}\left(\kappa ; u ; \Theta^{x}, \Theta^{y}\right) \varphi\right](x):=} \\
& \quad \frac{1}{(2 \pi \varepsilon)^{3 d / 2}} \int_{\mathbb{R}^{3 d}} e^{\frac{i}{\varepsilon} \Phi^{\kappa}\left(x, y, q, p ; \Theta^{x}, \Theta^{y}\right)}\left(L_{y}^{\dagger}\right)^{n}[u(x, y, q, p) \varphi(y)] d q d p d y,
\end{aligned}
$$

where

- $\Theta^{x}$ and $\Theta^{y}$ are complex symmetric matrices (i.e. $\Theta=\Theta^{\dagger}$ ) with positive definite real part,

- the complex-valued phase-function is given by

$$
\begin{aligned}
\Phi^{\kappa}\left(x, y, q, p ; \Theta^{x}, \Theta^{y}\right)= & S^{\kappa}(q, p)-p \cdot(y-q)+\Xi^{\kappa}(q, p) \cdot\left(x-X^{\kappa}(q, p)\right) \\
& +\frac{i}{2}(y-q) \cdot \Theta^{y}(y-q) \\
& +\frac{i}{2}\left(x-X^{\kappa}(q, p)\right) \cdot \Theta^{x}\left(x-X^{\kappa}(q, p)\right)
\end{aligned}
$$

- and the differential operator $L_{y}$ is defined by

$$
L_{y}=\frac{1}{1+\left|\nabla_{y} \Phi^{\kappa}\left(x, y, q, p ; \Theta^{x}, \Theta^{y}\right)\right|^{2}}\left[1-i \varepsilon \nabla_{y} \overline{\Phi^{\kappa}\left(x, y, q, p ; \Theta^{x}, \Theta^{y}\right)} \cdot \nabla_{y}\right] \text {. }
$$

The operator $L_{y}^{\dagger}$, the Banach-space adjoint of $L_{y}$, i.e.

$$
\int_{\mathbb{R}^{d}} v(y)\left[L_{y}^{\dagger} u\right](y) d y=\int_{\mathbb{R}^{d}}\left[L_{y} v\right](y) u(y) d y,
$$

makes the integrals absolutely convergent. Moreover, it is constructed such that expression (16) coincides for different $n$ with $n>m^{p}+d$ (especially with $n=0$, if $\left.m^{p}<-d\right)$.

The following theorem combines the central results of RoSw07. 


\section{Theorem 1.}

1. If $u \in S[+\infty ; 4 d], \mathcal{I}^{\varepsilon}\left(\kappa ; u ; \Theta^{x}, \Theta^{y}\right)$ sends $\mathcal{S}\left(\mathbb{R}^{d}, \mathbb{C}\right)$ into itself and is continuous.

2. If $u \in S[0 ; 4 d], \mathcal{I}^{\varepsilon}\left(\kappa ; u ; \Theta^{x}, \Theta^{y}\right)$ can be extended in a unique way to a linear bounded operator $L^{2}\left(\mathbb{R}^{d}, \mathbb{C}\right) \rightarrow L^{2}\left(\mathbb{R}^{d}, \mathbb{C}\right)$ and there exists a constant $C\left(M_{0}^{\kappa}, \Theta^{x}, \Theta^{y}\right)$ such that

$$
\left\|\mathcal{I}^{\varepsilon}\left(\kappa ; u ; \Theta^{x}, \Theta^{y}\right)\right\|_{L^{2} \rightarrow L^{2}} \leq C\left(M_{0}^{\kappa} ; \Theta^{x}, \Theta^{y}\right) \sum_{|\alpha| \leq 4 d+1}\left\|\partial_{(x, y)}^{\alpha} u\right\|_{L^{\infty}} .
$$

In the special case where $u \in S[0 ; 2 d]$ is independent of $(x, y)$, we have

$$
\left\|\mathcal{I}^{\varepsilon}\left(\kappa ; u ; \Theta^{x}, \Theta^{y}\right)\right\|_{L^{2} \rightarrow L^{2}} \leq 2^{-d / 2} \operatorname{det}\left(\Re \Theta^{x} \Re \Theta^{y}\right)^{-\frac{1}{4}}\|u\|_{L^{\infty}} .
$$

Remark 3.

1. The dependence of $C\left(M_{0}^{\kappa} ; \Theta^{x}, \Theta^{y}\right)$ on $M_{0}^{\kappa}, \Theta^{x}$ and $\Theta^{y}$ can be made more explicit. Consider RoSw07 for the precise expression.

2. There is an analogous result for Weyl-quantised pseudodifferential operators

$$
\left(o p^{\varepsilon}(h) \psi\right)(x):=\frac{1}{(2 \pi \varepsilon)^{d}} \int_{T^{*} \mathbb{R}} e^{\frac{i}{\varepsilon} \xi \cdot(x-y)} h\left(\frac{x+y}{2}, \xi\right) \psi(y) d y d \xi,
$$

see for example Ma02:

(a) If $h \in S[+\infty ; 2 d], o p^{\varepsilon}(h)$ sends $\mathcal{S}\left(\mathbb{R}^{d}, \mathbb{C}\right)$ into itself and is continuous.

(b) If $h \in S[0 ; 2 d], o p^{\varepsilon}(h)$ can be extended in a unique way to a linear bounded operator $L^{2}\left(\mathbb{R}^{d}, \mathbb{C}\right) \rightarrow L^{2}\left(\mathbb{R}^{d}, \mathbb{C}\right)$ with $\varepsilon$-independent norm

$$
\left\|o p^{\varepsilon}(h)\right\|_{L^{2} \rightarrow L^{2}} \leq C \sum_{|\alpha| \leq 2 d+1}\left\|\partial_{(x, \xi)}^{\alpha} h\right\|_{L^{\infty}} .
$$

The second part is the famous Calderón-Vaillancourt Theorem.

3. We have $\mathcal{I}^{\varepsilon}\left(\mathrm{id} ; \operatorname{det}\left(\Theta^{x}+\Theta^{y}\right)^{\frac{1}{2}} ; \Theta^{x}, \Theta^{y}\right)=\mathrm{id}$, compare the appendix for the correct choice of the square root.

\section{Composition with PDOs and time-derivatives}

The standard approach in the field of asymptotic analysis consists in a two step procedure. First, one constructs an asymptotic solution $U_{N}^{\varepsilon}(t, s) \psi_{s}^{\varepsilon}$ of order $O\left(\varepsilon^{N+1}\right)$, i.e. a function which fulfills

$$
\left(i \varepsilon \frac{d}{d t}-H^{\varepsilon}(t)\right) U_{N}^{\varepsilon}(t, s) \psi_{s}^{\varepsilon}=\varepsilon^{N+1} R_{N}^{\varepsilon}(t, s) \psi_{s}^{\varepsilon} .
$$

If one can establish an $\varepsilon$-independent bound on the remainder $R_{N}^{\varepsilon}(t, s)$, the asymptotic solution can be turned into an approximate solution of the unitary group with help of a special version of Gronwall's Lemma, (see for example Lemma 2.8 in Ha98 for the strategy of the proof): 
Lemma 1. Let $U^{\varepsilon}(t, s)$ be the propagator of the time-dependent Schrödingerequation

$$
\left(i \varepsilon \frac{d}{d t}-H^{\varepsilon}(t)\right) \psi^{\varepsilon}(t)=0, \quad \psi^{\varepsilon}(s)=\psi_{s}^{\varepsilon} \in D \subset L^{2}\left(\mathbb{R}^{d}, \mathbb{C}\right)
$$

for some family of self-adjoint operators $H^{\varepsilon}(t)$ with common domain D. Moreover, for some $T>0$ and $-T \leq t, s \leq T$ let $U_{N}^{\varepsilon}(t, s)$ be a family of bounded operators, which is strongly differentiable with respect to $t$, leaves the domain of $H^{\varepsilon}(t)$ invariant and which fulfills

$$
i \varepsilon \frac{d}{d t} U_{N}^{\varepsilon}(t, s) \psi^{\varepsilon}(s)-H^{\varepsilon}(t) U_{N}^{\varepsilon}(t, s) \psi^{\varepsilon}(s)=\varepsilon^{N+1} R_{N}^{\varepsilon}(t, s) \psi^{\varepsilon}(s)
$$

with $U_{N}^{\varepsilon}(s, s)=$ id. If $\left\|R_{N}^{\varepsilon}(t, s)\right\|_{L^{2} \rightarrow L^{2}}<\infty$ for all $-T \leq t, s \leq T$, we have

$$
\left\|U_{N}^{\varepsilon}(t, s)-U^{\varepsilon}(t, s)\right\|_{L^{2} \rightarrow L^{2}} \leq \varepsilon^{N}\left|\int_{s}^{t}\left\|R_{N}^{\varepsilon}(\tau, s)\right\|_{L^{2} \rightarrow L^{2}} d \tau\right| .
$$

In this section, we state the intermediate results needed for the construction of the asymptotic solution.

In Proposition 2, we show using Weyl-quantisation that the composition of differential operators with Fourier Integral Operators is again an FIO. Moreover, we give an asymptotic expansion of the symbol of the new FIO, whose terms but for the last are $x$-independent. This is important, as $x$-dependence of the symbol may be converted to $\varepsilon$-dependence, which can be seen from Lemma 3 . Proposition 3 deals with the time-derivative of a family of FIOs. Finally, we will establish an uniqueness result for symbols and canonical transformations in Proposition 4

\subsection{Statement of intermediate results}

To state our results, we need the matrix $\mathcal{Z}(q, p)=Z_{z}^{\kappa}(q, p)\left(\Theta^{x}\right)^{\frac{1}{2}}$, which already appeared as $\mathcal{Z}\left(F^{\kappa}(q, p)\right)$ in the statement of our main result in the introduction. We justify this abuse of notation by better readability of the formulae presented here. The invertibility of $\mathcal{Z}(q, p)$, which is implicitly claimed in the following statements, is shown in Lemma 2 .

The composition result reads:

Proposition 2. Let $h \in S\left[m_{h} ; 2 d\right]$ be polynomial in $\xi$ and $u \in S\left[m_{u} ; 2 d\right]$. Then we have

$o p^{\varepsilon}(h) \mathcal{I}^{\varepsilon}\left(\kappa ; u ; \Theta^{x}, \Theta^{y}\right)=\mathcal{I}^{\varepsilon}\left(\kappa ; \sum_{n=0}^{N} \varepsilon^{n} v_{n} ; \Theta^{x}, \Theta^{y}\right)+\varepsilon^{N+1} \mathcal{I}^{\varepsilon}\left(\kappa ; v_{N+1}^{\varepsilon} ; \Theta^{x}, \Theta^{y}\right)$

as operators from $\mathcal{S}\left(\mathbb{R}^{d}, \mathbb{C}\right)$ to $\mathcal{S}\left(\mathbb{R}^{d}, \mathbb{C}\right)$.

$v_{n} \in S\left[m_{u}+m_{h} ; 2 d\right], n \leq N$ and $v_{N+1}^{\varepsilon} \in S\left[\left(m_{h}, m_{u}+m_{h}\right) ;(d, 2 d)\right]$ are given by

$$
\begin{aligned}
v_{n}(q, p) & =L_{n}\left[h ; \kappa ; \Theta^{x}, \Theta^{y}\right] u(q, p) \\
v_{N+1}^{\varepsilon}(x, q, p) & =L_{N+1}^{\varepsilon}\left[h ; \kappa ; \Theta^{x}, \Theta^{y}\right] u(q, p),
\end{aligned}
$$


where $L_{n}\left[h ; \kappa ; \Theta^{x}, \Theta^{y}\right]$ and $L_{N+1}^{\varepsilon}\left[h ; \kappa ; \Theta^{x}, \Theta^{y}\right]$ are linear differential operators of order $n$ and $N+1$ in $(q, p)$. The coefficients of the $L_{n}\left[h ; \kappa ; \Theta^{x}, \Theta^{y}\right]$ are rational functions, with a numerator depending on derivatives of $h$ from order $n$ to $2 n$ and derivatives of $F^{\kappa}(q, p)$ of order $\leq n$, and a denominator of the form $\operatorname{det}(\mathcal{Z}(q, p))^{m}$ for some $m>0$. $L_{N}^{\varepsilon}\left[h ; \kappa ; \Theta^{x}, \Theta^{y}\right]$ is of the same form depending on derivatives of $h$ from order $N$ to $2 N+1$ and derivatives of $F^{\kappa}(q, p)$ of order $\leq 2 N+1$.

The explicit expressions for $v_{0}, v_{1}$ and $v_{2}$ are

$$
\begin{aligned}
& \begin{aligned}
v_{0}(q, p)= & u(q, p)(h \circ \kappa)(q, p) \\
v_{1}(q, p)= & -\operatorname{div}_{z}\left(\left(\left(h_{x}+i \Theta^{x} h_{\xi}\right) \circ \kappa(q, p)\right)^{\dagger} \mathcal{Z}^{-1}(q, p) u(q, p)\right) \\
& +u(q, p) \frac{1}{2} \operatorname{tr}\left(\mathcal{Z}^{-1}(q, p) \partial_{z}\left(\left(h_{x}+i \Theta^{x} h_{\xi}\right) \circ \kappa(q, p)\right)\right)
\end{aligned} \\
& \begin{aligned}
v_{2}(q, p)= & L_{2}\left[h_{\geq 3} ; \kappa ; \Theta^{x}, \Theta^{y}\right] u(q, p) \\
+ & \left.\frac{1}{2} \sum_{k=1}^{d} \operatorname{div}_{z}\left(u(q, p) \partial_{z_{k}}\left[\left(\left(\partial_{x}+i \Theta^{x} \partial_{\xi}\right)^{2} h\right) \circ \kappa(q, p) \mathcal{Z}^{-1}(q, p) e_{k}\right)\right]^{\dagger} \mathcal{Z}^{-1}(q, p)\right)
\end{aligned}
\end{aligned}
$$

The linear partial differential operator $L_{2}\left[h_{\geq 3} ; \kappa ; \Theta^{x}, \Theta^{y}\right]$ depends on derivatives of $h$ of order 3 and 4.

Remark 4. As the coefficients of the differential operators are rational functions of the form described in the statement, a bound for the elements of $F^{\kappa}(q, p)$ and their derivatives of the form $C \varepsilon^{-\rho}$ gives a bound for the coefficients of the form $C^{\prime} \varepsilon^{-M \rho}$ for some $M \in \mathbb{N}$, where $C^{\prime}$ depends on derivatives of $h$.

The second result of this section will investigate the time-derivative of a family of FIOs. In the case of a time-dependent family of canonical transformations, we have the following result:

Proposition 3. Let $u \in C\left(\mathbb{R}, S\left[\left(m_{q}, m_{p}\right) ;(d, d)\right]\right)$ be a family of time-dependent symbols with $u(\cdot, q, p) \in C^{1}(\mathbb{R}, \mathbb{C})$ and $\left(\frac{d}{d t} u\right)(t, \cdot, \cdot) \in S\left[\left(m_{q}, m_{p}\right) ;(d, d)\right], \kappa^{t} a$ family of canonical transformations of class $\mathcal{B}, S^{\kappa^{t}}$ an action associated to $\kappa^{t}$, $\Theta^{x} \in C^{1}(\mathbb{R}, \mathrm{Gl}(d))$ a family of complex symmetric matrices with positive definite real part and $\Theta^{y}$ complex symmetric with positive definite real part. We have

$$
i \varepsilon \frac{d}{d t} \mathcal{I}^{\varepsilon}\left(\kappa^{t} ; u ; \Theta^{x}(t), \Theta^{y}\right)=\mathcal{I}^{\varepsilon}\left(\kappa^{t} ; \sum_{n=0}^{2} \varepsilon^{n} v_{n} ; \Theta^{x}(t), \Theta^{y}\right)
$$


with

$$
\begin{aligned}
v_{0}(t, q, p)= & u(t, q, p)\left(-\frac{d}{d t} S^{\kappa^{t}}(q, p)+\frac{d}{d t} X^{\kappa^{t}}(q, p) \cdot \Xi^{\kappa^{t}}(q, p)\right) \\
v_{1}(t, q, p)= & i \frac{d}{d t} u(t, q, p) \\
& +\operatorname{div}_{z}\left(\left(\frac{d}{d t} \Xi^{\kappa^{t}}(q, p)-i \Theta^{x}(t) \frac{d}{d t} X^{\kappa^{t}}(q, p)\right)^{\dagger} \mathcal{Z}^{-1}(t, q, p) u(t, q, p)\right) \\
& -\frac{i}{2} u(t, q, p) \operatorname{tr}\left(\mathcal{Z}^{-1}(t, q, p) X_{z}^{\kappa}(q, p) \frac{d}{d t} \Theta^{x}(t)\right) \\
v_{2}(t, q, p)= & -\sum_{k=1}^{d} \operatorname{div}_{z}\left(\partial_{z_{k}}\left(\frac{d}{d t} \Theta^{x}(t) \mathcal{Z}^{-1}(t, q, p) e_{k} u(q, p)\right)^{\dagger} \mathcal{Z}^{-1}(t, q, p)\right)
\end{aligned}
$$

where $v_{0}, v_{1}, v_{2} \in C^{0}\left(\mathbb{R}, S\left[\left(m_{q}, m_{p}\right) ;(d, d)\right]\right)$.

Remark 5. In both propositions, the case of a linear canonical transformation, a quadratic symbol $h$ and a constant symbol $u$ results in $v_{n}=0$ for $n \geq 2$. This will result in the exactness of the Herman-Kluk expression for quadratic Hamiltonians.

Finally, we have the following uniqueness result.

Proposition 4. Let $\kappa_{1}$ and $\kappa_{2}$ be two canonical transformations of class $\mathcal{B}$ and $u, v \in S[0 ; 2 d]$. If

$$
\lim _{\varepsilon \rightarrow 0}\left\|\mathcal{I}^{\varepsilon}\left(\kappa_{1} ; u ; \Theta^{x}, \Theta^{y}\right)-\mathcal{I}^{\varepsilon}\left(\kappa_{2} ; v ; \Theta^{x}, \Theta^{y}\right)\right\|_{L^{2} \rightarrow L^{2}}=0,
$$

then $u=v$ and $\kappa_{1}(q, p)=\kappa_{2}(q, p)$ for all $(q, p) \in \operatorname{supp} u$.

\subsection{Some auxiliary results}

The proofs of these results will strongly rely on results about conversion of $x$-dependence to $\varepsilon$-dependence. We start with the following auxiliary result.

Lemma 2. We have

$i \Phi_{z}^{\kappa}\left(x, y, q, p ; \Theta^{x}, \Theta^{y}\right)=Z_{z}^{\kappa}(q, p)\left(\Theta^{x}\right)^{\frac{1}{2}}\left(x-X^{\kappa}(q, p)\right)=: \mathcal{Z}(q, p)\left(x-X^{\kappa}(q, p)\right)$.

$\mathcal{Z}(q, p)=\left(i\left(\Theta^{y}\right)^{-1} \quad \mathrm{id}\right)\left(F^{\kappa}(q, p)\right)^{\dagger}\left(-i \Theta^{x} \quad \mathrm{id}\right)^{\dagger}$ is invertible and its inverse $\mathcal{Z}^{-1}(q, p)$ is in the class $S[0 ; 2 d]$. 
Remark 6.

1. We recall that

$$
Z_{z}^{\kappa}(q, p)=\left(\left(\Theta^{y}\right)^{-1} \partial_{q}-i \partial_{p}\right)\left(\left(\Theta^{x}\right)^{\frac{1}{2}} X^{\kappa}(q, p)+i\left(\Theta^{x}\right)^{-\frac{1}{2}} \Xi^{\kappa}(q, p)\right) .
$$

2. Obviously, $\mathcal{Z}(q, p)$ depends on $q$ and $p$ only via the elements of $F^{\kappa}(q, p)$. For better readability, we do not explicitly denote this dependence. Moreover, we drop the dependence on $\Theta^{x}$ and $\Theta^{y}$ in the notation.

3. For a linear canonical transformation $\kappa(q, p)=M\left(\begin{array}{l}q \\ p\end{array}\right)$, with $M \in \operatorname{Sp}(2 d)$, we have $F^{\kappa}(q, p)=M$, so $\mathcal{Z}(q, p)=\left(i\right.$ id $\left.\quad\left(\Theta^{y}\right)^{-1}\right) M^{\dagger}\left(-i \Theta^{x} \quad \text { id }\right)^{\dagger}$ is constant with respect to $(q, p)$.

Proof. The derivatives of $\Phi^{\kappa}\left(x, y, q, p ; \Theta^{x}, \Theta^{y}\right)$ with respect to $q$ and $p$ are

$$
\begin{aligned}
& \Phi_{q}^{\kappa}\left(x, y, q, p ; \Theta^{x}, \Theta^{y}\right)=\left[\Xi_{q}^{\kappa}-i X_{q}^{\kappa} \Theta^{x}\right](q, p)\left(x-X^{\kappa}(q, p)\right)-i \Theta^{y}(y-q) \\
& \Phi_{p}^{\kappa}\left(x, y, q, p ; \Theta^{x}, \Theta^{y}\right)=\left[\Xi_{p}^{\kappa}-i X_{p}^{\kappa} \Theta^{x}\right](q, p)\left(x-X^{\kappa}(q, p)\right)-(y-q),
\end{aligned}
$$

which gives the identity for $\mathcal{Z}(q, p)$. Obviously, $\mathcal{Z}(q, p)$ inherits its symbol class from $F^{\kappa}(q, p)$. Moreover, we have

$$
\begin{aligned}
& \mathcal{Z}(q, p)\left(\Re \Theta^{x}\right)^{-1} \mathcal{Z}(q, p)^{*} \\
= & 2 \Re\left(\Theta^{y}\right)^{-1}+\left(\Lambda\left(\Theta^{x}\right) F^{\kappa}(q, p)\left(\begin{array}{c}
i\left(\Theta^{y}\right)^{-1} \\
-\mathrm{id}
\end{array}\right)\right)^{*}\left(\Lambda\left(\Theta^{x}\right) F^{\kappa}(q, p)\left(\begin{array}{c}
i\left(\Theta^{y}\right)^{-1} \\
-\mathrm{id}
\end{array}\right)\right)
\end{aligned}
$$

with

$$
\Lambda(\Theta)=\left(\begin{array}{cc}
(\Re \Theta)^{1 / 2} & 0 \\
(\Re \Theta)^{-1 / 2} \Im \Theta & (\Re \Theta)^{-1 / 2}
\end{array}\right) .
$$

Hence, by the superadditivity of the determinant for positive definite hermitian matrices, $\operatorname{det} \mathcal{Z}(q, p)$ is uniformly bounded away from 0 for all $q$ and $p$, so by its expression via the formula of minors, $\mathcal{Z}^{-1}(q, p) \in S[0 ; 2 d]$, as $\mathcal{Z}(q, p)$ is.

We introduce the following notation:

Definition 7. Two symbols $u, v \in S[+\infty ; 4 d]$ are said to be equivalent with respect to $\kappa$ if

$$
\mathcal{I}^{\varepsilon}\left(\kappa ; u ; \Theta^{x}, \Theta^{y}\right)=\mathcal{I}^{\varepsilon}\left(\kappa ; v ; \Theta^{x}, \Theta^{y}\right)
$$

as operators from $\mathcal{S}\left(\mathbb{R}^{d}, \mathbb{C}\right)$ to $\mathcal{S}\left(\mathbb{R}^{d}, \mathbb{C}\right)$. In this case we write $u \sim v$.

The central technical identity, on which most of the following results rely, is contained in the following lemma, which is a special case of Lemma 5 in RoSw07. Here we present a proof based on definition [6] whereas in [RoSw07, an alternative definition based on a smoothing of the oscillatory integrals is used.

Lemma 3. Let $u \in S\left[\left(m^{x}, m^{q}, m^{p}\right) ;(d, d, d)\right]$. Then

$$
\left(x_{j}-X_{j}^{\kappa}(q, p)\right) u \sim \varepsilon v,
$$

where $v \in S\left[\left(m^{x}, m^{q}, m^{p}\right) ;(d, d, d)\right]$ is given by

$$
v(x, q, p)=-\operatorname{div}_{z}\left(e_{j}^{\dagger} \mathcal{Z}^{-1}(q, p) u(x, q, p)\right)
$$


Proof. We introduce a countable, locally finite partition of unity $\chi_{l}(q, p)$ with $\sup _{N, q, p}\left|\sum_{l=0}^{N} \partial_{z_{k}} \chi_{l}(q, p)\right|=C<\infty$. Let $m>m^{p}+d$. Using dominated convergence, we get

$$
\begin{aligned}
\mathcal{I}^{\varepsilon}\left(\kappa ;\left(x_{j}-X_{j}^{\kappa}(q, p)\right) u ; \Theta^{x}, \Theta^{y}\right) \varphi(x) \\
=\frac{1}{(2 \pi \varepsilon)^{3 d / 2}} \sum_{l=0}^{\infty} \int_{\mathbb{R}^{3 d}} \chi_{l}(q, p)\left(x_{j}-X_{j}^{\kappa}(q, p)\right) e^{\frac{i}{\varepsilon} \Phi^{\kappa}}\left(L_{y}^{\dagger}\right)^{m}(u \varphi)(x, y, q, p) d q d p d y \\
=\frac{1}{(2 \pi \varepsilon)^{3 d / 2}} \sum_{l=0}^{\infty} \int_{\mathbb{R}^{3 d}} \chi_{l}(q, p)\left(x_{j}-X_{j}^{\kappa}(q, p)\right) e^{\frac{i}{\varepsilon} \Phi^{\kappa}}(u \varphi)(x, y, q, p) d q d p d y \\
=\frac{-\varepsilon}{(2 \pi \varepsilon)^{3 d / 2}} \sum_{l=0}^{\infty} \int_{\mathbb{R}^{3 d}} \chi_{l}(q, p) e^{\frac{i}{\varepsilon} \Phi^{\kappa}} \sum_{k=1}^{d} \partial_{z_{k}}\left(\mathcal{Z}_{j k}^{-1} u\right)(x, q, p) \varphi(y) d q d p d y \\
-\frac{\varepsilon}{(2 \pi \varepsilon)^{3 d / 2}} \sum_{l=0}^{\infty} \int_{\mathbb{R}^{3 d}} \sum_{k=1}^{d}\left(\partial_{z_{k}} \chi_{l}\right)(q, p) e^{\frac{i}{\varepsilon} \Phi^{\kappa}}\left(\mathcal{Z}_{j k}^{-1} u \varphi\right)(x, y, q, p) d q d p d y
\end{aligned}
$$

After introducing $m$ powers of $L_{y}^{\dagger}$, the integrand in (27) is dominated by

$$
C \sum_{k=1}^{d}\left|\mathcal{Z}_{j k}^{-1}(q, p) e^{\frac{i}{\varepsilon} \Phi^{\kappa}}\left(L_{y}^{\dagger}\right)^{m}(u \varphi)(x, y, q, p)\right| .
$$

Thus (27) vanishes, whereas (26) is the expected quantity.

By iterative applications, the previous lemma easily extends to any polynomial $x$ dependence. We will only state the result for quadratic polynomials and refer the reader to the proof of Proposition 2 where a similar problem is treated, for the general case.

Proposition 5. Let $P(q, p, x)=\alpha(q, p)+a(q, p) \cdot x+x \cdot A(q, p) x$ be a quadratic polynomial with coefficients in $S\left[m_{P} ; 2 d\right]$ and $u \in S\left[m_{u} ; 2 d\right]$. Then

$$
P\left(q, p, x-X^{\kappa}(q, p)\right) u(q, p) \quad \sim \quad \sum_{n=0}^{2} \varepsilon^{n} v_{n}(q, p),
$$

where the $v_{n} \in S\left[m_{P}+m_{u} ; 2 d\right]$ are given by

$$
\begin{aligned}
v_{0}(q, p)= & \alpha(q, p) u(q, p) \\
v_{1}(q, p)= & -\operatorname{div}_{z}\left(a(q, p)^{\dagger} \mathcal{Z}^{-1}(q, p) u(q, p)\right) \\
& +\operatorname{tr}\left(\mathcal{Z}^{-1}(q, p) X_{z}^{\kappa}(q, p) A(q, p)\right) u(q, p) \\
v_{2}(q, p)= & \sum_{k=1}^{d} \operatorname{div}_{z}\left(\partial_{z_{k}}\left[A(q, p) \mathcal{Z}^{-1}(q, p) e_{k} u(q, p)\right]^{\dagger} \mathcal{Z}^{-1}(q, p)\right)
\end{aligned}
$$

Proof. The statement follows from two successive applications of Lemma 3 , The 
quadratic part contributes to $v_{1}$ and $v_{2}$ :

$$
\begin{aligned}
& \left(x-X^{\kappa}(q, p)\right) \cdot A(q, p)\left(x-X^{\kappa}(q, p)\right) u(q, p) \\
\sim & -\varepsilon \operatorname{div}_{z}\left(u(q, p)\left(x-X^{\kappa}(q, p)\right)^{\dagger} A(q, p) \mathcal{Z}^{-1}(q, p)\right) \\
= & -\varepsilon \operatorname{tr}\left(\partial_{z}\left[u(q, p)\left(x-X^{\kappa}(q, p)\right)^{\dagger} A(q, p) \mathcal{Z}^{-1}(q, p)\right]\right) \\
= & \varepsilon \operatorname{tr}\left(A(q, p) \mathcal{Z}^{-1}(q, p) X_{z}^{\kappa}(q, p)\right) u(q, p) \\
& \quad-\varepsilon \sum_{k=1}^{d}\left(x-X^{\kappa}(q, p)\right)^{\dagger}\left(\partial_{z_{k}}\left(A(q, p) \mathcal{Z}^{-1}(q, p) e_{k} u(q, p)\right)\right) \\
\sim & \varepsilon \operatorname{tr}\left(\mathcal{Z}^{-1}(q, p) X_{z}^{\kappa}(q, p) A(q, p)\right) u(q, p) \\
& \quad+\varepsilon^{2} \sum_{k=1}^{d} \operatorname{div}_{z}\left[\partial_{z_{k}}\left(A(q, p) \mathcal{Z}^{-1}(q, p) e_{k} u(q, p)\right)^{\dagger} \mathcal{Z}^{-1}(q, p)\right],
\end{aligned}
$$

where we used the invariance of the trace under cyclic permutations.

\subsection{Proofs of the Propositions 24}

We have now established all auxiliary results necessary for proving our central propositions

Proof. (of Proposition 2) Let $\varphi \in \mathcal{S}\left(\mathbb{R}^{d}, \mathbb{C}\right)$. The composition of $o p^{\varepsilon}(h)$ with the FIO applied to $\varphi$ is

$$
\begin{aligned}
& {\left[o p^{\varepsilon}(h) \mathcal{I}^{\varepsilon}\left(\kappa ; u ; \Theta^{x}, \Theta^{y}\right) \varphi\right](x) } \\
= & \frac{1}{(2 \pi \varepsilon)^{5 d / 2}} \int_{\mathbb{R}^{5 d}} h\left(\frac{x+w}{2}, \xi\right) e^{\frac{i}{\varepsilon} \Psi^{\kappa}\left(x, w, y, q, p ; \Theta^{x}, \Theta^{y}\right)} u(q, p) \varphi(y) d q d p d y d w d \xi,
\end{aligned}
$$

where $\Psi^{\kappa}\left(x, w, y, q, p ; \Theta^{x}, \Theta^{y}\right):=\xi \cdot(x-w)+\Phi^{\kappa}\left(w, y, q, p ; \Theta^{x}, \Theta^{y}\right)$.

We introduce the following combinations of positions and momenta, which correspond to creation and annihilation "variables"

$$
\begin{aligned}
& a(x, \xi):=\left(\Theta^{x}\right)^{\frac{1}{2}} x+i\left(\Theta^{x}\right)^{-\frac{1}{2}} \xi, \\
& \bar{a}(x, \xi):=\left(\Theta^{x}\right)^{\frac{1}{2}} x-i\left(\Theta^{x}\right)^{-\frac{1}{2}} \xi
\end{aligned}
$$

and their "dual operators"

$$
\begin{aligned}
\partial_{a} & :=\frac{1}{2}\left(\left(\Theta^{x}\right)^{-\frac{1}{2}} \partial_{x}-i\left(\Theta^{x}\right)^{\frac{1}{2}} \partial_{\xi}\right) \\
\partial_{\bar{a}} & :=\frac{1}{2}\left(\left(\Theta^{x}\right)^{-\frac{1}{2}} \partial_{x}+i\left(\Theta^{x}\right)^{\frac{1}{2}} \partial_{\xi}\right) .
\end{aligned}
$$

The Taylor-expansion of the symbol $h$ to order $2 N$ around $\kappa(q, p)$ reads

$$
\begin{aligned}
h(x, \xi)= & \sum_{|\alpha+\beta| \leq 2 N} \frac{1}{\alpha ! \beta !}\left(\left(\partial_{a}^{\alpha} \partial_{\bar{a}}^{\beta} h\right) \circ \kappa\right)(q, p)\left(a-Z^{\kappa}(q, p)\right)^{\alpha}\left(\bar{a}-\bar{Z}^{\kappa}(q, p)\right)^{\beta} \\
& +\sum_{|\alpha+\beta|=2 N+1}\left(a-Z^{\kappa}(q, p)\right)^{\alpha}\left(\bar{a}-\bar{Z}^{\kappa}(q, p)\right)^{\beta} R_{\alpha, \beta}(a, \bar{a}, q, p) \\
= & h_{\mathrm{T}}\left(a-Z^{\kappa}(q, p), \bar{a}-\bar{Z}^{\kappa}(q, p)\right)+h_{\mathrm{R}}(a, \bar{a}, q, p),
\end{aligned}
$$


where

$$
\begin{aligned}
& R_{\alpha, \beta}(a, \bar{a}, q, p) \\
= & \frac{|\alpha+\beta|}{\alpha ! \beta !} \int_{0}^{1} \sigma^{|\alpha+\beta|-1}\left(\partial_{a}^{\alpha} \partial_{\bar{a}}^{\beta} h\right)\left(x+\sigma\left(X^{\kappa}(q, p)-x\right), \xi+\sigma\left(\Xi^{\kappa}(q, p)-\xi\right)\right) d \sigma .
\end{aligned}
$$

We split the integral into a part which contains the Taylor polynomial $h_{\mathrm{T}}$ and a remainder containing $h_{R}$. In the first step, we discuss only the part containing $h_{\mathrm{T}}$. We have, dropping the argument $\left(\frac{x+w}{2}, \xi\right)$ of $a$ and $\bar{a}$,

$$
\left(\frac{1}{2}\left(\Theta^{x}\right)^{\frac{1}{2}} \partial_{\xi}-i\left(\Theta^{x}\right)^{-\frac{1}{2}} \partial_{w}\right) \Psi^{\kappa}=a-Z^{\kappa}(q, p) .
$$

Moreover

$$
\begin{aligned}
& \left(\frac{1}{2}\left(\Theta^{x}\right)^{\frac{1}{2}} \partial_{\xi}-i\left(\Theta^{x}\right)^{-\frac{1}{2}} \partial_{w}\right)\left(a-Z^{\kappa}(q, p)\right)=0 \\
& \left(\frac{1}{2}\left(\Theta^{x}\right)^{\frac{1}{2}} \partial_{\xi}-i\left(\Theta^{x}\right)^{-\frac{1}{2}} \partial_{w}\right)\left(\bar{a}-\bar{Z}^{\kappa}(q, p)\right)=-i \text { id. }
\end{aligned}
$$

By integration by parts we get

$$
\left(a-Z^{\kappa}(q, p)\right)^{\alpha}\left(\bar{a}-\bar{Z}^{\kappa}(q, p)\right)^{\beta} v(q, p) \sim \frac{\varepsilon^{|\alpha|} \beta !}{(\beta-\alpha) !}\left(\bar{a}-\bar{Z}^{\kappa}(q, p)\right)^{\beta-\alpha} v(q, p),
$$

where we extended the meaning of " " in an obvious way. We have

$$
\begin{aligned}
& \left(\frac{1}{2}\left(\Theta^{x}\right)^{\frac{1}{2}} \partial_{\xi}+i\left(\Theta^{x}\right)^{-\frac{1}{2}} \partial_{w}+2 i\left(\Theta^{x}\right)^{\frac{1}{2}} \mathcal{Z}^{-1}(q, p) \partial_{z}\right)\left(\bar{a}-\bar{Z}^{\kappa}(q, p)\right) \\
= & -2 i\left(\Theta^{x}\right)^{\frac{1}{2}} \mathcal{Z}^{-1}(q, p) \partial_{z} \bar{Z}^{\kappa}(q, p)
\end{aligned}
$$

and hence

$$
\begin{aligned}
& \left(\bar{a}-\bar{Z}^{\kappa}(q, p)\right)^{\gamma} v(q, p) \\
\sim & -\frac{2 \varepsilon}{\# \gamma} \sum_{k \mid \gamma_{k} \neq 0} \operatorname{div}_{z}\left(e_{k}^{\dagger}\left(\Theta^{x}\right)^{\frac{1}{2}} \mathcal{Z}^{-1}(q, p)\left(\bar{a}-\bar{Z}^{\kappa}(q, p)\right)^{\gamma-e_{k}} v(q, p)\right) \\
= & \frac{\varepsilon}{\# \gamma} \sum_{k \mid \gamma_{k} \neq 0}\left(\sum_{m=1}^{d}\left(\gamma-e_{k}\right)_{m}\left(\bar{a}-\bar{Z}^{\kappa}(q, p)\right)^{\gamma-e_{k}-e_{m}}\left(\mathcal{L}_{\left(e_{k}, e_{m}\right)} v\right)(q, p)\right. \\
& \left.+\left(\bar{a}-\bar{Z}^{\kappa}(q, p)\right)^{\gamma-e_{k}}\left(\mathcal{L}_{e_{k}} v\right)(q, p)\right)
\end{aligned}
$$

where the differential operators $\mathcal{L}_{\left(e_{k}, e_{m}\right)}$ and $\mathcal{L}_{e_{k}}$ are given by

$$
\begin{aligned}
\left(\mathcal{L}_{\left(e_{k}, e_{m}\right)} v\right)(q, p) & :=2 e_{k}^{\dagger}\left(\Theta^{x}\right)^{\frac{1}{2}} \mathcal{Z}^{-1}(q, p) \partial_{z} \bar{Z}^{\kappa} e_{m} v(q, p) \\
\left(\mathcal{L}_{e_{k}} v\right)(q, p) & :=-2 \operatorname{div}_{z}\left(e_{k}^{\dagger}\left(\Theta^{x}\right)^{\frac{1}{2}} \mathcal{Z}^{-1}(q, p) v(q, p)\right)
\end{aligned}
$$


and $\# \gamma$ denotes the number of non-zero components of $\gamma$.

The symmetrization by the summation over $k$ allows for the iteration of the procedure. We define the three sets

$$
\Gamma_{1}:=\left\{\gamma \in \mathbb{N}^{d}|| \gamma \mid=1\right\}, \quad \Gamma_{2}:=\Gamma_{1} \times \Gamma_{1}, \quad \Gamma:=\Gamma_{1} \cup \Gamma_{2} .
$$

In expression (29) the sum is taken over all possible reductions of the multiindex $\gamma$ by elements of the "brick-sets" $\Gamma_{1}$ and $\Gamma_{2}$. After another integration by parts in all terms with $\left(\bar{a}-\bar{Z}^{\kappa}(q, p)\right)$-dependence, the sum is taken over all possible reductions of $\gamma$ by elements in $\Gamma \times \Gamma$, which may be considered as a two-step path in $\Gamma$, plus the terms which already led to $\gamma=0$ in the first step. So after the removal of all $\left(\bar{a}-\bar{Z}^{\kappa}(q, p)\right)$-dependence, the sum is taken over all possible paths in the "brick-set" $\Gamma$ which reduce $\gamma$ to zero. To formalise this idea, we define the map

$$
\begin{aligned}
& {[\cdot] \quad: \quad \Gamma \rightarrow \mathbb{N}^{d}} \\
& {[\gamma]:= \begin{cases}\gamma & \gamma \in \Gamma_{1} \\
\gamma_{1}+\gamma_{2} & \gamma=\left(\gamma_{1}, \gamma_{2}\right) \in \Gamma_{2} .\end{cases} }
\end{aligned}
$$

With

$$
\lambda\left(\gamma, \gamma_{1}, \ldots, \gamma_{n}\right)= \begin{cases}\left(\#\left(\gamma-\sum_{l<n}\left[\gamma_{l}\right]\right)\right)^{-1} & \gamma_{n} \in \Gamma_{1} \\ \left(\#\left(\gamma-\sum_{l<n}\left[\gamma_{l}\right]\right)\right)^{-1}\left(\gamma-\sum_{l<n}\left[\gamma_{l}\right]-e_{j}\right)_{k} & \gamma_{n}=\left(e_{j}, e_{k}\right) \in \Gamma_{2}\end{cases}
$$

we have

$$
\begin{aligned}
& \left(\bar{a}-\bar{Z}^{\kappa}(q, p)\right)^{\gamma} v(q, p) \\
\sim & \sum_{\substack{\gamma_{1} \ldots, \gamma_{k} \in \Gamma \\
\left[\gamma_{1}\right]+\ldots+\left[\gamma_{k}\right]=\gamma}} \varepsilon^{k} \lambda\left(\gamma, \gamma_{1}, \ldots, \gamma_{k}\right) \ldots \lambda\left(\gamma, \gamma_{1}, \gamma_{2}\right) \lambda\left(\gamma, \gamma_{1}\right)\left(\mathcal{L}_{\gamma_{k}} \ldots \mathcal{L}_{\gamma_{1}} v\right)(q, p) .
\end{aligned}
$$

Combining (28) and (30), we get

$$
\begin{aligned}
& \left(h_{T} u\right)(q, p) \\
\sim & \sum_{n=0}^{N} \varepsilon^{n} L_{n}\left[h ; \kappa ; \Theta^{x}, \Theta^{y}\right] u(q, p)+\varepsilon^{N+1} \widetilde{L}_{N+1}^{\varepsilon}\left[h ; \kappa ; \Theta^{x}, \Theta^{y}\right] u(q, p) \\
= & \sum_{\substack{|\beta| \leq 2 N \\
\alpha \leq \beta}} \sum_{\substack{\gamma_{1} \ldots, \gamma_{k} \in \Gamma \\
\left[\gamma_{1}\right] \ldots+\left[\gamma_{k}\right]=\beta-\alpha}} \frac{\varepsilon^{|\alpha|+k}}{\alpha !(\beta-\alpha) !}\left(\prod_{l=1}^{k} \lambda\left(\gamma, \gamma_{1}, \ldots, \gamma_{l}\right) \mathcal{L}_{\gamma_{l}}\right)\left(u \partial_{a}^{\alpha} \partial_{\bar{a}}^{\beta} h \circ \kappa\right)(q, p) \\
\quad & +\varepsilon^{N+1} \widetilde{L}_{N+1}^{\varepsilon}\left[h ; \kappa ; \Theta^{x}, \Theta^{y}\right] u(q, p)
\end{aligned}
$$

where $\varepsilon^{N+1} \widetilde{L}_{N+1}^{\varepsilon}\left[h ; \kappa ; \Theta^{x}, \Theta^{y}\right] u(q, p)$ contains all the terms of order $\varepsilon^{N+1}$ and higher. As $k$ ranges between $\lceil|\beta-\alpha| / 2\rceil$ and $|\beta-\alpha|$, we have

$$
\begin{aligned}
& L_{n}\left[h ; \kappa ; \Theta^{x}, \Theta^{y}\right] \\
& =\sum_{\substack{n \leq|\alpha+\beta| \leq 2 n \\
\alpha \leq \beta}} \sum_{\substack{\gamma_{1}, \ldots, \gamma_{n-|\alpha|} \in \Gamma \\
\left[\gamma_{1}\right]+\ldots+\left[\gamma_{n}-|\alpha|\right]=\beta-\alpha}} \frac{1}{\alpha !(\beta-\alpha) !}\left(\prod_{l=1}^{n-|\alpha|} \lambda\left(\gamma, \gamma_{1}, \ldots, \gamma_{l}\right) \mathcal{L}_{\gamma_{l}}\right)
\end{aligned}
$$


with the following convention for $n-|\alpha|=0$

$$
\sum_{\gamma_{1}, \ldots, \gamma_{n-|\alpha|}} \prod_{l=1}^{n-|\alpha|} \lambda\left(\gamma, \gamma_{1}, \ldots, \gamma_{l}\right) \mathcal{L}_{\gamma_{l}}=\mathrm{id} .
$$

For the first few terms in the expansion, we have more transparent expressions. The zeroth order term

$$
(h \circ \kappa)(q, p) u(q, p)
$$

is provided by $\alpha=\beta=0$. For the first order term, there are three contributions.

1. The terms with $|\beta|=1, \alpha=\beta$, which result in

$$
\varepsilon \operatorname{tr}\left(\left(\left(\partial_{a} \partial_{\bar{a}} h\right) \circ \kappa\right)(q, p)\right) u(q, p) .
$$

2. The terms $|\beta|=1, \alpha=0$, which give

$$
-\varepsilon \operatorname{div}_{z}\left(\left(\left(\partial_{\bar{a}} h\right)^{\dagger} \circ \kappa\right)(q, p)\left(\Theta^{x}\right)^{\frac{1}{2}} \mathcal{Z}^{-1}(q, p) u(q, p)\right) .
$$

3. The first order contribution of terms $|\beta|=2, \alpha=0$, which is

$$
\varepsilon \operatorname{tr}\left(\mathcal{Z}^{-1}(q, p) \partial_{z} \bar{Z}^{\kappa}(q, p)\left(\left(\operatorname{Hess}_{\bar{a}} h\right) \circ \kappa\right)(q, p)\left(\Theta^{x}\right)^{\frac{1}{2}}\right) u(q, p) .
$$

By an application of the chain rule, they may be combined to

$$
\begin{aligned}
= & -\varepsilon \operatorname{div}_{z}\left(\left(\left(\partial_{\bar{a}} h\right)^{\dagger} \circ \kappa\right)(q, p)\left(\Theta^{x}\right)^{\frac{1}{2}} \mathcal{Z}^{-1}(q, p) u(q, p)\right) \\
& +\varepsilon \operatorname{tr}\left(\mathcal{Z}^{-1}(q, p) \partial_{z}\left(\left(\Theta^{x}\right)^{\frac{1}{2}}\left(\left(\partial_{\bar{a}} h\right) \circ \kappa\right)(q, p)\right)\right) u(q, p) .
\end{aligned}
$$

The second order term arises in a similar way.

The form of the coefficients of the differential operators $L_{n}\left[h ; \kappa ; \Theta^{x}, \Theta^{y}\right]$ follows, if $\mathcal{Z}^{-1}(q, p)$ is expressed by the formula of minors. With respect to the symbol class of $v_{n}$, it is sufficient to note that $\kappa \in S[1 ; 2 d]$.

We turn to the discussion of the remainder. The $\left(a-Z^{\kappa}(q, p)\right)$ and $\left(\bar{a}-\bar{Z}^{\kappa}(q, p)\right)$ factors may be converted to $\varepsilon$-dependence analogously to $h_{T}$, resulting in terms of order $\varepsilon^{N+1}$ to $\varepsilon^{2 N+2}$. As $h(x, \xi)$ is polynomial in $\xi$, the resulting expression equals the application of a differential operator of order $m_{\xi}$ to an FIO. We have $\partial_{x} \Phi^{\kappa}\left(x, y, q, p ; \Theta^{x}, \Theta^{y}\right)=\Xi^{\kappa}(q, p)+i \Theta^{x}\left(x-X^{\kappa}(q, p)\right)$, hence the symbol class by iterative applications of Lemma 3

Remark 7. In the case $\kappa=\mathrm{id}, u(q, p)=1, \Theta^{x}=\Theta^{y}=\mathrm{id}$, the proof provides the asymptotic expansion of the Anti-Wick symbol of a Weyl quantised pseudodifferential operator.

We have $\mathcal{Z}(q, p)=2 \mathrm{id}, Z^{\kappa}=q+i p$ and $\bar{Z}^{\kappa}=q-i p$. Hence $\partial_{z} \bar{Z}^{\kappa}=0$, $\mathcal{L}_{\left(e_{j}, e_{k}\right)}=0$ and $\left(\mathcal{L}_{e_{j}} u\right)(q, p)=-\left(\partial_{z_{j}} u\right)(q, p)$. Moreover, all $\mathcal{L}_{e_{j}}$ commute.

By straightforward calculation, the Anti-Wick symbol is

$$
\sum_{|\beta|=0}^{N} \frac{(-1)^{|\beta|} \varepsilon^{|\beta|}}{\beta !}\left(\Delta^{\beta} h\right)(q, p),
$$


where

$$
\Delta^{\beta}=\prod_{k=1}^{d}\left(\partial_{x_{k}}^{2}+\partial_{\xi_{k}}^{2}\right)^{\beta_{k}} .
$$

Thus formally

$$
h_{\mathrm{AW}}=e^{-\varepsilon \Delta} h_{\mathrm{Weyl}},
$$

so we recover that the Anti-Wick quantisation is the solution of the Cauchyproblem for the inverse heat-equation at time $t=\varepsilon$ with the Weyl-symbol as initial datum (compare $\mathrm{Ma02}$, where the transition is expressed by the heatkernel).

Next, we give the easy proof of Proposition 3

Proof. (of Proposition 3) By direct computation $i \varepsilon \frac{d}{d t} \mathcal{I}^{\varepsilon}\left(\kappa^{t} ; u\right)$ is an FIO with symbol

$$
\begin{aligned}
i \varepsilon \frac{d}{d t} u(t, q, p) & -u(t, q, p) \frac{d}{d t} \Phi^{\kappa^{t}}\left(x, y, q, p ; \Theta^{x}(t), \Theta^{y}\right) \\
=i \varepsilon \frac{d}{d t} u(t, q, p) & -u(t, q, p)\left[\frac{d}{d t} S^{\kappa^{t}}(q, p)-\frac{d}{d t} X^{\kappa^{t}}(q, p) \cdot \Xi^{\kappa^{t}}(q, p)\right. \\
+ & \left(\frac{d}{d t} \Xi^{\kappa^{t}}(q, p)-i\left(\Theta^{x}(t) \frac{d}{d t} X^{\kappa^{t}}(q, p)\right)\right) \cdot\left(x-X^{\kappa^{t}}(q, p)\right) \\
+ & \left.\frac{i}{2}\left(x-X^{\kappa^{(t, s)}}(q, p)\right) \cdot \frac{d}{d t} \Theta^{x}(t)\left(x-X^{\kappa^{(t, s)}}(q, p)\right)\right]
\end{aligned}
$$

The expressions (23) and (24) follow from Proposition 5

We close this section with the proof of Proposition 4

Proof. (of Proposition 4) The proof relies on the inner product

$$
\left\langle g_{\kappa^{\prime}\left(q_{0}, p_{0}\right)}^{\varepsilon, \overline{\Theta^{x}}}, \mathcal{I}^{\varepsilon}\left(\kappa ; w ; \Theta^{x}, \Theta^{y}\right) g_{\left(q_{0}, p_{0}\right)}^{\varepsilon, \Theta^{y}}\right\rangle
$$

for symbols $w \in S[0 ; 2 d]$ and canonical transformations $\kappa, \kappa^{\prime}$ of class $\mathcal{B}$, where

$$
g_{(q, p)}^{\varepsilon, \Theta}(x)=\frac{\operatorname{det}(\Re \Theta)^{\frac{1}{4}}}{(\pi \varepsilon)^{d / 4}} e^{-(x-q) \cdot \Theta(x-q) / 2 \varepsilon} e^{i p \cdot(x-q) / \varepsilon} .
$$

Straightforward calculation gives

$$
\text { (31) }=\frac{2^{d}}{(\pi \varepsilon)^{d}} \frac{\operatorname{det}\left(\Re \Theta^{x}\right)^{\frac{1}{4}} \operatorname{det}\left(\Re \Theta^{y}\right)^{\frac{1}{4}}}{\operatorname{det}\left(2 \Theta^{x}\right)^{\frac{1}{2}} \operatorname{det}\left(2 \Theta^{y}\right)^{\frac{1}{2}}} \int e^{\frac{i}{\varepsilon} \Psi^{\kappa, \kappa^{\prime}}\left(q_{0}, p_{0}, q, p\right)} w(q, p) d q d p,
$$

where

$$
\begin{aligned}
& \Psi^{\kappa, \kappa^{\prime}}\left(q, p, q_{0}, p_{0}\right)=S^{\kappa}(q, p) \\
& +\frac{1}{2}\left(q-q_{0}\right)\left(p+p_{0}\right)-\frac{1}{2}\left(X^{\kappa}(q, p)-X^{\kappa^{\prime}}\left(q_{0}, p_{0}\right)\right)\left(\Xi^{\kappa}(q, p)+\Xi^{\kappa^{\prime}}\left(q_{0}, p_{0}\right)\right) \\
& +i\left(q_{0}-q\right) \cdot \Theta^{y}\left(q_{0}-q\right) / 4+i\left(p_{0}-p\right) \cdot\left(\Theta^{y}\right)^{-1}\left(p_{0}-p\right) / 4 \\
& +i\left(X^{\kappa^{\prime}}\left(q_{0}, p_{0}\right)-X^{\kappa}(q, p)\right) \cdot \Theta^{x}\left(X^{\kappa^{\prime}}\left(q_{0}, p_{0}\right)-X^{\kappa}(q, p)\right) / 4 \\
& +i\left(\Xi^{\kappa^{\prime}}\left(q_{0}, p_{0}\right)-\Xi^{\kappa}(q, p)\right) \cdot\left(\Theta^{x}\right)^{-1}\left(\Xi^{\kappa^{\prime}}\left(q_{0}, p_{0}\right)-\Xi^{\kappa}(q, p)\right) / 4
\end{aligned}
$$


We chose $\sigma \in C_{0}^{\infty}\left(\mathbb{R}^{2 d}, \mathbb{R}\right)$ with $\sigma=1$ in an neighborhood of $\left(q_{0}, p_{0}\right)$ and split the integral into

$$
\begin{aligned}
& \left\langle g_{\kappa^{\prime}\left(q_{0}, p_{0}\right)}^{\varepsilon, \overline{\Theta^{x}}}, \mathcal{I}^{\varepsilon}\left(\kappa ; \sigma w ; \Theta^{x}, \Theta^{y}\right) g_{\kappa\left(q_{0}, p_{0}\right)}^{\varepsilon, \Theta^{y}}\right\rangle \\
+ & \left\langle g_{\kappa^{\prime}\left(q_{0}, p_{0}\right)}^{\varepsilon, \mathcal{I}^{x}}, \mathcal{I}^{\varepsilon}\left(\kappa ;(1-\sigma) w ; \Theta^{x}, \Theta^{y}\right) g_{\kappa\left(q_{0}, p_{0}\right)}^{\varepsilon, \Theta^{y}}\right\rangle .
\end{aligned}
$$

It is easily seen that

$$
\Im \Psi\left(q, p, q_{0}, p_{0}\right)=0 \text { and }\left(\nabla_{(q, p)} \Re \Psi\right)\left(q, p, q_{0}, p_{0}\right)=0
$$

if and only if $(q, p)=\left(q_{0}, p_{0}\right)$ and $\kappa\left(q_{0}, p_{0}\right)=\kappa^{\prime}\left(q_{0}, p_{0}\right)$. Thus the phase in (33) is non-stationary on the support of $w(1-\sigma)$, so after integrations by parts with the operator

$$
\frac{-i \varepsilon}{\left\|\nabla_{(q, p)} \Psi\left(q_{0}, p_{0}, q, p\right)\right\|^{2}} \overline{\nabla_{(q, p)} \Psi\left(q_{0}, p_{0}, q, p\right)} \cdot \nabla_{(q, p)}
$$

we have $\lim _{\varepsilon \rightarrow 0}(33)=0$. By the same argument, the case $\kappa\left(q_{0}, p_{0}\right) \neq \kappa^{\prime}\left(q_{0}, p_{0}\right)$ gives $\lim _{\varepsilon \rightarrow 0}(32)=0$. In the case $\kappa\left(q_{0}, p_{0}\right)=\kappa^{\prime}\left(q_{0}, p_{0}\right)$, we have

$$
\operatorname{Hess}_{\left(q_{0}, p_{0}\right)} \Psi^{\kappa, \kappa}=\frac{i}{2}\left(\begin{array}{cc}
\Theta^{y} & 0 \\
0 & \left(\Theta^{y}\right)^{-1}
\end{array}\right)+\frac{i}{2} F^{\kappa}\left(q_{0}, p_{0}\right)^{\dagger}\left(\begin{array}{cc}
\Theta^{x} & 0 \\
0 & \left(\Theta^{x}\right)^{-1}
\end{array}\right) F^{\kappa}\left(q_{0}, p_{0}\right),
$$

at the stationary points, so by the Stationary Phase Theorem (Theorem 7.7.5. in $\mathrm{Hö83}$ )

$$
\lim _{\varepsilon \rightarrow 0}\left\langle g_{\kappa\left(q_{0}, p_{0}\right)}^{\varepsilon, \overline{\Theta^{x}}}, \mathcal{I}^{\varepsilon}\left(\kappa ; \sigma w ; \Theta^{x}, \Theta^{y}\right) g_{\kappa\left(q_{0}, p_{0}\right)}^{\varepsilon, \Theta^{y}}\right\rangle=C\left[\kappa ; \Theta^{x} ; \Theta^{y}\right] w\left(q_{0}, p_{0}\right),
$$

with the non-vanishing constant

$$
\begin{aligned}
C\left[\kappa ; \Theta^{x} ; \Theta^{y}\right]=2^{2 d} \frac{\operatorname{det}\left(\Re \Theta^{x}\right)^{\frac{1}{4}}\left(\Re \Theta^{y}\right)^{\frac{1}{4}}}{\operatorname{det}\left(\Theta^{x}\right)^{\frac{1}{2}} \operatorname{det}\left(\Theta^{y}\right)^{\frac{1}{2}}} \\
\quad \operatorname{det}\left(\left(\begin{array}{cc}
\Theta^{y} & 0 \\
0 & \left(\Theta^{y}\right)^{-1}
\end{array}\right)+F^{\kappa}\left(q_{0}, p_{0}\right)^{\dagger}\left(\begin{array}{cc}
\Theta^{x} & 0 \\
0 & \left(\Theta^{x}\right)^{-1}
\end{array}\right) F^{\kappa}\left(q_{0}, p_{0}\right)\right)^{-\frac{1}{2}} .
\end{aligned}
$$

Subsuming this discussion, we have

$$
\begin{aligned}
0 & =\lim _{\varepsilon \rightarrow 0}\left\langle g_{\kappa_{1}\left(\Theta_{0}, p_{0}\right)},\left[\mathcal{I}^{\varepsilon}\left(\kappa_{1} ; u ; \Theta^{x}, \Theta^{y}\right)-\mathcal{I}^{\varepsilon}\left(\kappa_{2} ; v ; \Theta^{x}, \Theta^{y}\right)\right] g_{\kappa_{1}\left(q_{0}, p_{0}\right)}^{\varepsilon, \Theta^{y}}\right\rangle \\
& = \begin{cases}C\left[\kappa_{1} ; \Theta^{x} ; \Theta^{y}\right] u\left(q_{0}, p_{0}\right) & \kappa_{1}\left(q_{0}, p_{0}\right) \neq \kappa_{2}\left(q_{0}, p_{0}\right) \\
C\left[\kappa_{1} ; \Theta^{x} ; \Theta^{y}\right] u\left(q_{0}, p_{0}\right)-C\left[\kappa_{2} ; \Theta^{x} ; \Theta^{y}\right] v\left(q_{0}, p_{0}\right) & \kappa_{1}\left(q_{0}, p_{0}\right)=\kappa_{2}\left(q_{0}, p_{0}\right)\end{cases}
\end{aligned}
$$

In the case $\kappa_{1}\left(q_{0}, p_{0}\right) \neq \kappa_{2}\left(q_{0}, p_{0}\right)$ we immediately get $u\left(q_{0}, p_{0}\right)=0$ and by symmetry $v\left(q_{0}, p_{0}\right)=0=u\left(q_{0}, p_{0}\right)$. In the case $\kappa_{1}\left(q_{0}, p_{0}\right)=\kappa_{2}\left(q_{0}, p_{0}\right)$, we either have $u\left(q_{0}, p_{0}\right)=0$ or $u\left(q_{0}, p_{0}\right) \neq 0$. In the first case, we immediately get $v\left(q_{0}, p_{0}\right)=0=u\left(q_{0}, p_{0}\right)$. In the second case $u$ does not vanish in a neighbourhood of $\left(q_{0}, p_{0}\right)$. Hence $\kappa_{1}=\kappa_{2}$ in the same neighborhood, thus $C\left[\kappa_{1} ; \Theta^{x} ; \Theta^{y}\right]=C\left[\kappa_{2} ; \Theta^{x} ; \Theta^{y}\right]$ and so $u\left(q_{0}, p_{0}\right)=v\left(q_{0}, p_{0}\right)$. 


\section{Uniform approximations of the unitary group}

In this section we will combine the results of Section 3.1 to our main result.

Theorem 2. Let $U^{\varepsilon}(t, s)$ be the propagator associated to the time-dependent Schrödinger-equation

$$
i \varepsilon \frac{d}{d t} \psi^{\varepsilon}(t)=H^{\varepsilon}(t) \psi^{\varepsilon}(t), \quad \psi^{\varepsilon}(s)=\psi_{s}^{\varepsilon} \in L^{2}\left(\mathbb{R}^{d}, \mathbb{C}\right)
$$

on the time-interval $-T \leq s, t \leq T$ where $H^{\varepsilon}(t)=o p^{\varepsilon}\left(h_{0}+\varepsilon h_{1}\right)$ with subquadratic $h_{0}(t, x, \xi)$ and sublinear $h_{1}(t, x, \xi)$, both polynomial in $\xi$. Moreover let

- $\Theta^{y} \in \operatorname{Gl}(d)$ be complex symmetric with positive definite real part and

- $\Theta^{x} \in C^{1}(\mathbb{R}, \mathrm{Gl}(d))$ be complex symmetric fulfilling $0<\gamma$ id $\leq \Re \Theta^{x}(t) \leq$ $\gamma^{\prime}$ id for all $t \in[-T, T]$ in the sense of quadratic forms.

Then

$$
\sup _{-T \leq s, t \leq T}\left\|U^{\varepsilon}(t, s)-\mathcal{I}^{\varepsilon}\left(\kappa^{(t, s)} ; \sum_{n=0}^{N} \varepsilon^{n} u_{n} ; \Theta^{x}(t), \Theta^{y}\right)\right\|_{L^{2} \rightarrow L^{2}} \leq C(T) \varepsilon^{N+1},
$$

where $\kappa^{(t, s)}$ and the $u_{n}$ are uniquely given as

- the Hamiltonian flow associated to $h_{0}$ and

- the solutions of

$$
\begin{aligned}
& \frac{d}{d t} u_{n}(t, s, q, p)=\frac{1}{2} u_{n}(t, s, q, p) \times \\
& \quad\left[\operatorname{tr}\left(\mathcal{Z}^{-1}(t, s, q, p) \frac{d}{d t} \mathcal{Z}(t, s, q, p)\right)-i h_{1}\left(t, X^{\kappa^{(t, s)}}(q, p), \Xi^{\kappa^{(t, s)}}(q, p)\right)\right] \\
& -\sum_{k=1}^{d} \operatorname{div}_{z}\left[\left(\partial_{z_{k}}\left(\frac{d}{d t} \Theta^{x}(t) \mathcal{Z}^{-1}(t, s, q, p) e_{k} u_{n-1}\right)\right)^{\dagger} \mathcal{Z}^{-1}(t, s, q, p)\right] \\
& +i \sum_{j=2}^{n-2} L_{j}\left[h_{0}(t) ; \kappa^{(t, s)} ; \Theta^{x}(t), \Theta^{y}\right] u_{n+1-j} \\
& +i \sum_{j=1}^{n-3} L_{j}\left[h_{1}(t) ; \kappa^{(t, s)} ; \Theta^{x}(t), \Theta^{y}\right] u_{n-j}
\end{aligned}
$$

with initial conditions

$$
\begin{aligned}
& u_{0}(s, s, q, p)=\operatorname{det}\left(\Theta^{x}(s)+\Theta^{y}\right)^{1 / 2} \\
& u_{n}(s, s, q, p)=0, \quad n \geq 1 .
\end{aligned}
$$

Corollary 1. Under the additional assumption

$$
\sum_{2 \leq|\alpha| \leq 4 d+7}\left\|\left(\partial_{(q, p)}^{\alpha} h\right)(t, q, p)\right\|_{L^{\infty}\left(\mathbb{R} \times \mathbb{R}^{2 d}\right)}<\infty,
$$


we have the following result on the Ehrenfest timescale $T(\varepsilon)=C_{T} \log \left(\varepsilon^{-1}\right)$ :

$$
\sup _{-T(\varepsilon) \leq s, t \leq T(\varepsilon)}\left\|U^{\varepsilon}(t, s)-\mathcal{I}^{\varepsilon}\left(\kappa^{(t, s)} ; u_{0} ; \Theta^{x}(t), \Theta^{y}\right)\right\| \leq C\left(C_{T}\right) \varepsilon^{1-\rho\left(C_{T}\right)},
$$

where $\rho\left(C_{T}\right)$ can be made arbitrary small, if $C_{T}$ is chosen small enough.

\section{Remark 8.}

1. We recall that $\mathcal{Z}(t, s, q, p)=\left(i\left(\Theta^{y}\right)^{-1} \mathrm{id}\right) F^{\kappa^{(t, s)}}(q, p)^{\dagger}\left(-i \Theta^{x}(t) \mathrm{id}\right)^{\dagger}$, thus the dependence of $\mathcal{Z}(t, s, q, p)$ on $q$ and $p$ is only via the Jacobian of the flow.

2. The expression for the leading order symbol is

$$
u_{0}(t, s, q, p)=\left(\operatorname{det} \Theta^{y} \mathcal{Z}(t, s, q, p)\right)^{\frac{1}{2}} e^{-i \int_{s}^{t} h_{1}\left(\tau, \kappa^{(\tau, s)}(q, p)\right) d \tau},
$$

where the branch of the square root is defined by continuity in $t$ starting from $u_{0}(s, s, q, p)=\operatorname{det}\left(\Theta^{x}(s)+\Theta^{y}\right)^{\frac{1}{2}}$, compare Remark 3. The corresponding FIO is known as the Herman-Kluk propagator in the chemical literature. Notice that the dependence of $u$ on $q$ and $p$ is only via $F^{\kappa^{(t, s)}}(q, p)$. Likewise, the $(q, p)$-dependence in $u_{k}$ is only via $F^{\kappa^{(t, s)}}(q, p)$ and its derivatives with respect to $q$ and $p$.

3. As an easy corollary we get that the FIO defined in the last theorem approximately inherits the properties of $U(t, s)$, i.e. it is almost unitary in the sense that

$$
\begin{aligned}
& \left\|\mathcal{I}^{\varepsilon}\left(\kappa^{(t, s)} ; u ; \Theta^{x}(t), \Theta^{y}\right) \mathcal{I}^{\varepsilon}\left(\kappa^{(t, s)} ; u ; \Theta^{x}(t), \Theta^{y}\right)^{*}-\mathrm{id}\right\| \leq C_{N} \varepsilon^{N+1} \\
& \left\|\mathcal{I}^{\varepsilon}\left(\kappa^{(t, s)} ; u ; \Theta^{x}(t), \Theta^{y}\right)^{*} \mathcal{I}^{\varepsilon}\left(\kappa^{(t, s)} ; u ; \Theta^{x}(t), \Theta^{y}\right)-\mathrm{id}\right\| \leq C_{N} \varepsilon^{N+1},
\end{aligned}
$$

where $u=\sum_{n=0}^{N} \varepsilon^{n} u_{n}$ and it almost fulfills the group property, i.e.

$$
\left\|\mathcal{I}^{\varepsilon}\left(\kappa^{\left(t, t^{\prime}\right)} ; u ; \mathrm{id}, \mathrm{id}\right) \mathcal{I}^{\varepsilon}\left(\kappa^{\left(t^{\prime}, s\right)} ; u ; \mathrm{id}, \mathrm{id}\right)-\mathcal{I}^{\varepsilon}\left(\kappa^{(t, s)} ; u ; \mathrm{id}, \mathrm{id}\right)\right\| \leq C_{N}^{\prime} \varepsilon^{N+1} .
$$

The result also holds for general $\Theta^{x}$ and $\Theta^{y}$. The possibility of stating the correct dependence of the symbol on the matrices is left to the reader.

4. In the case of a linear flow

$$
\kappa^{(t, s)}(q, p)=M(t, s)\left(\begin{array}{l}
q \\
p
\end{array}\right)
$$

the approximation becomes exact as the symbols $v_{n}$ in Propositions 2 and 3 vanish for $n \geq 2$. This can be rephrased as

$$
\mathcal{I}^{\varepsilon}\left(\kappa^{(t, s)} ; u_{0} ; \Theta^{x}, \Theta^{y}\right)=\operatorname{Met}^{\varepsilon}\left(F^{\kappa^{(t, s)}}(q, p)\right)
$$

where $\mathrm{Met}^{\varepsilon}$ denotes the $\varepsilon$-dependent metaplectic representation. 
5. The Ehrenfest result does not generalise to the higher order corrections. This is in accordance with the situation in BaGrPa99, where holomorphicity of the symbol is required to get higher order versions of Egorov's Theorem on the Ehrenfest timescale.

6. The proof will produce the following byproduct: the so-called Frozen Gaussian Approximation, which is obtained by still choosing $\kappa^{(t, s)}$ as the Hamiltonian flow but keeping $u_{0}$ constant for all $q, p$ and $t$, is an asymptotic solution of the Schrödinger equation of order $O(\varepsilon)$. Thus it approximates the unitary group for the short times of order $\varepsilon$. It will not be a valid approximation for longer times because of the uniqueness of the symbol.

Proof. By Theorem 1, an FIO associated to a $C^{1}$ family $\kappa^{(t, s)}$ of canonical transformation of class $\mathcal{B}$ and $(x, y)$-independent symbol $u=\sum_{n=0}^{N} \varepsilon^{n} u_{n}, u_{n} \in$ $C^{1}(\mathbb{R}, S[0 ; 2 d])$ leaves $\mathcal{S}\left(\mathbb{R}^{d}, \mathbb{C}\right)$ invariant. Thus, we can plug such an operator as an ansatz into the time-dependent Schrödinger equation (11). By Propositions 2 and 3 we have a representation

$$
\begin{aligned}
& \left(i \varepsilon \frac{d}{d t}-o p^{\varepsilon}\left(h_{0}+\varepsilon h_{1}\right)\right) \mathcal{I}^{\varepsilon}\left(\kappa^{(t, s)} ; \sum_{n=0}^{N} \varepsilon^{n} u_{n} ; \Theta^{x}(t), \Theta^{y}\right) \\
= & \mathcal{I}^{\varepsilon}\left(\kappa^{(t, s)} ; \sum_{n=0}^{N+1} \varepsilon^{n} v_{n} ; \Theta^{x}(t), \Theta^{y}\right)+\varepsilon^{N+2} \mathcal{I}^{\varepsilon}\left(\kappa^{(t, s)} ; v_{N+2}^{\varepsilon} ; \Theta^{x}(t), \Theta^{y}\right)
\end{aligned}
$$

on $\mathcal{S}\left(\mathbb{R}^{d}, \mathbb{C}\right)$. We will show that the $v_{n}, 0 \leq n \leq N+1$ vanish, if $\kappa^{(t, s)}$ and $u_{n} \in S[0,2 d], 0 \leq n \leq N$ are chosen properly. Moreover, it will turn out that $v_{N+2}^{\varepsilon}$ is of class $S[0 ; 3 d]$. Thus, by Theorem 1, $\mathcal{I}^{\varepsilon}\left(\kappa^{(t, s)} ; \sum_{n=0}^{N} \varepsilon^{n} u_{n} ; \Theta^{x}(t), \Theta^{y}\right)$ is an asymptotic solution of order $N+2$. The statement will then follow by Lemma 1 .

Combining Propositions 2 and 3 , one recognises $v_{0}$ as the product of $u_{0}$ and

$$
\left(-\frac{d}{d t} S^{\kappa^{(t, s)}}(q, p)+\frac{d}{d t} X^{\kappa^{(t, s)}}(q, p) \cdot \Xi^{\kappa^{(t, s)}}(q, p)-h_{0}\left(t, \kappa^{(t, s)}(q, p)\right)\right) .
$$

As we do not expect $\mathcal{I}^{\varepsilon}\left(\kappa ; 0 ; \Theta^{x}, \Theta^{y}\right)=0$ to be a good approximation of $U(t, s)$, we require (34) to vanish. By combining its derivatives with respect to $p$ and $q$, it is easily seen that this is the case if and only if $\kappa^{(t, s)}$ is the Hamiltonian flow associated to $h_{0}$.

There are several parts which contribute to $v_{1}$ :

1. the zeroth order terms of Propositions 2 and 3 applied to $u_{1}$,

2. the first order terms of Propositions 2 and 3 applied to $u_{0}$,

3. the zeroth order term of Proposition 2 applied to $u_{0}$ for the subprincipal symbol $h_{1}$. 
Thus we get the following expression for $v_{1}$ :

$$
\begin{aligned}
& u_{1}\left[-\frac{d}{d t} S^{\kappa^{(t, s)}}(q, p)+\frac{d}{d t} X^{\kappa^{(t, s)}}(q, p) \cdot \Xi^{\kappa^{(t, s)}}(q, p)-h_{0}\left(t, \kappa^{(t, s)}(q, p)\right)\right] \\
& +\operatorname{div}_{z}\left(\left[\left(\partial_{x} h_{0}+i \Theta^{x}(t) \partial_{\xi} h_{0}\right)\left(t, \kappa^{(t, s)}(q, p)\right)\right]^{\dagger} \mathcal{Z}^{-1}(t, s, q, p) u_{0}\right) \\
& +\operatorname{div}_{z}\left(\left(\frac{d}{d t} \Xi^{\kappa^{(t, s)}}(q, p)-i \Theta^{x}(t) \frac{d}{d t} X^{\kappa^{(t, s)}}(q, p)\right)^{\dagger} \mathcal{Z}^{-1}(t, s, q, p) u_{0}\right) \\
& -u_{0} \frac{1}{2} \operatorname{tr}\left(\mathcal{Z}^{-1}(t, s, q, p) \partial_{z}\left[\left(\partial_{x} h_{0}+i \Theta^{x}(t) \partial_{\xi} h_{0}\right)\left(t, \kappa^{(t, s)}(q, p)\right)\right]\right) \\
& +i \frac{d}{d t} u_{0}-\frac{i}{2} u_{0} \operatorname{tr}\left(\mathcal{Z}^{-1}(t, s, q, p) X_{z}^{\kappa}(q, p) \frac{d}{d t} \Theta^{x}(t)\right) \\
& -u_{0} h_{1}\left(t, \kappa^{(t, s)}(q, p)\right) \\
& =i \frac{d}{d t} u_{0}-\frac{i}{2} u_{0} \operatorname{tr}\left(\mathcal{Z}^{-1}(t, s, q, p) \frac{d}{d t} \mathcal{Z}(t, s, q, p)\right)-u_{0} h_{1}\left(t, \kappa^{(t, s)}(q, p)\right)
\end{aligned}
$$

as (35), (36) and (37) vanish because of the choice of $\kappa^{(t, s)}$ as the Hamilton flow. As the linearisation of $\operatorname{det}(A)$ is $\operatorname{det}(A) \operatorname{tr}\left(A^{-1} \mathrm{~d} A\right)$ for invertible $A$, the equation $v_{1}=0$ with initial conditions that recover identity, is solved by

$$
u_{0}(t, s, q, p)=\left(\operatorname{det}\left(\Theta^{y} \mathcal{Z}(t, s, q, p)\right)\right)^{\frac{1}{2}} \exp \left(-i \int_{s}^{t} h_{1}\left(\tau, \kappa^{(\tau, s)}(q, p)\right) d \tau\right),
$$

which is of class $S[0 ; 2 d]$.

$v_{n}$ is given by the following expression:

$$
\begin{aligned}
& i \frac{d}{d t} u_{n-1}-\frac{i}{2} u_{n-1} \operatorname{tr}\left(\mathcal{Z}^{-1}(t, s, q, p) \frac{d}{d t} \mathcal{Z}(t, s, q, p)\right)-u_{n-1} h_{1}\left(t, \kappa^{(t, s)}(q, p)\right) \\
& -\sum_{k=1}^{d} \operatorname{div}_{z}\left(\left(\partial_{z_{k}}\left(\frac{d}{d t} \Theta^{x}(t) \mathcal{Z}^{-1}(t, s, q, p) e_{k} u_{n-2}\right)\right)^{\dagger} \mathcal{Z}^{-1}(t, s, q, p)\right) \\
& -i \sum_{j=2}^{n-2} L_{j}\left[h_{0}(t) ; \kappa^{(t, s)} ; \Theta^{x}(t), \Theta^{y}\right] u_{n-j} \\
& -i \sum_{j=1}^{n-3} L_{j}\left[h_{1}(t) ; \kappa^{(t, s)} ; \Theta^{x}(t), \Theta^{y}\right] u_{n-j-1},
\end{aligned}
$$

where we already dropped the terms analogous to (35)-(37). The equation $v_{n}=0$ is easily solved by variation of the constant and has a solution in $S[0 ; 2 d]$. We finally note that the highest order symbol is of class $S[0 ; 3 d]$. Thus, we have established that the constructed FIO is an asymptotic solution of order $N+2$ on the class of Schwartz functions, so the result follows by the strategy outlined at the beginning of the proof.

We turn to the uniqueness. Assume that there are $\widetilde{\kappa}^{(t, s)}$ and $\widetilde{u} \in S[0 ; 2 d]$ such that

$$
\left\|U^{\varepsilon}(t, s)-\mathcal{I}^{\varepsilon}\left(\widetilde{\kappa}^{(t, s)} ; \widetilde{u} ; \Theta^{x}(t), \Theta^{y}\right)\right\| \leq C^{\prime}(T) \varepsilon .
$$


In this case we have

$$
\left\|\mathcal{I}^{\varepsilon}\left(\kappa^{(t, s)} ; u_{0} ; \Theta^{x}(t), \Theta^{y}\right)-\mathcal{I}^{\varepsilon}\left(\widetilde{\kappa}^{(t, s)} ; \widetilde{u} ; \Theta^{x}(t), \Theta^{y}\right)\right\| \leq\left(C(T)+C^{\prime}(T)\right) \varepsilon
$$

and thus we get $\widetilde{\kappa}^{(t, s)}=\kappa^{(t, s)}$ and $\widetilde{u}=u_{0}$ on $\operatorname{supp} u_{0}=\mathbb{R}^{2 d}$ by Proposition 4 , The uniqueness of the higher order corrections follows inductively by the same kind of argument.

Proof. (of Corollary 1) To extend the result to the Ehrenfest timescale, we have to study the dependence of the remainder's symbol $v_{2}^{\varepsilon}$ on $T=T(\varepsilon)$ and to show that the growth of

$$
\sum_{|\alpha| \leq 4 d+1}\left\|\partial_{x}^{\alpha} v_{2}^{\varepsilon}\right\|_{L^{\infty}}
$$

does not exceed $O(\varepsilon)$ in the $\varepsilon \rightarrow 0$ limit if $C_{T}$ is sufficiently small.

The dependence comes from the elements of $F^{\kappa^{(t, s)}}(q, p)$ and its derivatives. By (14), they allow for a bound of the form $C^{\prime}\left(C_{T}\right) e^{-\rho\left(C_{T}\right)}$, where $\rho\left(C_{T}\right)$ can be made arbitrary small if $C_{T}$ is chosen small enough. Moreover, $v_{2}^{\varepsilon}$ has polynomial growth in these quantities, which follows from the form of the differential operators of Proposition 2 the explicit expression of $u_{0}$ and the bound away from zero of the determinant of $\mathcal{Z}(t, s, q, p)$, compare the proof of Lemma 2 , Combining these facts, the result follows.

\section{A Gaussian integrals with non-real matrices}

We consider the convex cone $\mathcal{C}$ of complex symmetric matrices with positive definite real part. Every matrix of $\mathcal{C}$ is invertible with its spectrum included in the open half plane $\{z \mid \Re z>0\}$. It follows from matrix theory (see [JoOkRe01]) that each element of $\mathcal{C}$ admits an unique square root in $\mathcal{C}$. Furthermore, the square root of $M$ is given by the Dunford-Taylor integral (see [Ka66] I.§5.6)

$$
M^{1 / 2}=\frac{1}{2 \pi i} \int_{\Gamma} z^{1 / 2}(M-z)^{-1} d z
$$

where the integration path is a closed contour in the half-plane $\{z \mid \Re z>0\}$ making a turn around each eigenvalue in the positive direction and the value of $z^{1 / 2}$ is chosen so that it is positive for real positive $z$. As a consequence, the square root $M^{1 / 2}$ is an holomorphic function of $M$. If one considers the computation of the Gaussian integral

$$
\frac{1}{(2 \pi \varepsilon)^{d / 2}} \int_{\mathbb{R}^{d}} e^{-\frac{M}{2 \varepsilon} x \cdot x} d x,
$$

it is well-known that its value is given by $(\operatorname{det} M)^{1 / 2}=\operatorname{det}\left(M^{1 / 2}\right)$ for positive definite real symmetric $M$. From the above discussion, it directly follows that this property extends to any matrix $M \in \mathcal{C}$ (see Appendix A in [Fo89] or Section 3.4. in [Hö83 for an alternative explanation). 


\section{References}

[BaGrPa99] D. Bambusi, S. Graffi and T. Paul, Long time semiclassical approximation of quantum flows: a proof of the Ehrenfest time, Asympt. Anal., 21, pp. 149-160 (1999).

[BiRo01] J.-M. Bily and D. Robert, The Semi-classical Van-Vleck Formula. Application to the Aharonov-Bohm Effect, Graffi, Sandro (ed.) et al., Long time behaviour of classical and quantum systems, Proceedings of the Bologna APTEX international conference, Bologna, Italy, September 13-17, 1999, Singapore: World Scientific, Ser. Concr. Appl. Math., 1, pp.89-106 (2001).

[Bu02] J. Butler, Global $h$ Fourier integral operators with complex-valued phase functions, Bull. London Math. Soc., 34 (4), pp.479-489 (2002).

[Co68] J.D. Cole, Pertubation Methods in Applied Mathematics, Blaisdell Publishing Co., Waltham, Mass. (1968)

[CoRo97] M. Combescure and D. Robert, Semiclassical spreading of quantum wave packets and applications near unstable fixed points of the classical flow, Asympt. Anal. 14, pp. 377-404 (1997).

[Fo89] G.B. Folland, Harmonic Analysis in Phase Space, Annals of Mathematics Studies 122, Princeton University Press (1989).

[Fu79] D. Fujiwara, A construction of the fundamental solution for the Schrödinger equation, J. d'Analyses Math., 35, pp.41-96 (1979).

[Ha85] G. Hagedorn, Semiclassical quantum mechanics I : The $\hbar \rightarrow 0$ Limit for Coherent States. Commun. Math. Phys., 71, p. 77-93 (1980).

[Ha98] G. Hagedorn, Raising and Lowering Operators for Semiclassical Wave Packets, Ann. Phys., 269 (1), pp. 77-104 (1998).

[HaRoGr04] C. Harabati, J.M. Rost and F. Grossman, Long-time and unitarity properties of semiclassical initial value representations, J. Chem. Phys., 120 (1), pp. 16-30 (2004).

[He75] E.J. Heller, Time-dependent approach to semiclassical dynamics, $J$. Chem. Phys. 62(4), pp. 1544-1555 (1975).

[He81] E.J. Heller, Frozen Gaussians: a very simple semiclassical approximation, J. Chem. Phys. 75(6), pp. 2923-2931 (1981).

[HeKl84] M.F. Herman and E. Kluk, A semiclassical justification for the use of non-spreading wavepackets in dynamics calculations, Chem. Phys., 91 (1), pp.27-34 (1984).

[Hö83] L. Hörmander, The Analysis of Linear Partial Differential Operators I, Springer-Verlag, New York (1983).

[JoOkRe01] C.R. Johnson, Y. Okubo, R. Reams, Uniqueness of Matrix Square Roots and an Application, Lin. Alg. Appl., 323, pp.51-60 (2001). 
[Ka94] K. Kay, Integral expressions for the semi-classical time-dependent propagator, J. Chem. Phys., 100 (6), pp.4377-4392 (1994).

[Ka06] K. Kay, The Herman-Kluk approximation: Derivation and semiclassical corrections, Chem. Phys., 322, pp.3-12 (2006).

[Ka66] T. Kato, Perturbation Theory for Linear Operators, Springer-Verlag, New York (1966).

[Ki82] H. Kitada, A calculus of Fourier integral operators and the global fundamental solution for a Schrödinger equation, Osaka J. Math., 19, pp.863-900 (1982).

[KiKu81] H. Kitada and H. Kumano-Go, A family of Fourier Integral Operators and the fundamental solution for a Schrödinger equation, Osaka J. Math., 18, pp.291-360 (1981).

[LaSi00] A. Laptev and I.M. Sigal, Global Fourier Integral Operators and semiclassical asymptotics, Review of Math. Phys., 12 (5), pp.749-766 (2000).

[Ma02] A. Martinez, An Introduction to Semiclassical and Microlocal Analysis, Universitext, Springer-Verlag, New York (2002).

[RoSw07] V. Rousse, T. Swart, Global $L^{2}$-Boundedness Theorems for Semiclassical Fourier Integral Operators with Complex Phase, submitted, preprint is available online at http://arxiv.org/abs/0710.4200.

[Ta04] D. Tataru, Phase space transforms and microlocal analysis, in Phase space analysis of partial differential equations Vol. II (Pubbl. Cent. Ric. Mat. Ennio Giorgi, Scuola Norm. Sup. Pisa), pp.505-524 (2004). 